\title{
ON THE INDUCTIVE LIMIT OF DIRECT SUMS OF SIMPLE TAI ALGEBRAS
}

\author{
BO CUI, CHUNLAN JIANG, AND LIANGQING LI
}

\begin{abstract}
An ATAI (or ATAF, respectively) algebra, introduced in [Jiang1] (or in [Fa] respectively) is an inductive limit $\lim _{n \rightarrow \infty}\left(A_{n}=\bigoplus_{i=1} A_{n}^{i}, \phi_{n m}\right)$, where each $A_{n}^{i}$ is a simple separable nuclear TAI (or TAF) $\mathrm{C}^{*}$-algebra with UCT property. In [Jiang1], the second author classified all ATAI algebras by an invariant consisting orderd total K-theory and tracial state spaces of cut down algebras under an extra restriction that all element in $K_{1}(A)$ are torsion. In this paper, we remove this restriction, and obtained the classification for all ATAI algebras with the Hausdorffized algebraic $K_{1}$-group as an addition to the invariant used in [Jiang1]. The theorem is proved by reducing the class to the classification theorem of $\mathcal{A H D}$ algebras with ideal property which is done in [GJL1]. Our theorem generalizes the main theorem of [Fa] and [Jiang1] (see corollary 4.3).
\end{abstract}

\section{Introduction}

In [Lin2], Lin gave an abstract description of simple AH algebras (with no dimension growth) classified in [EGL1]. He described the decomposition property of simple AH algebras in [G3] as TAI property and proved that all simple separable nuclear TAI algebras with UCT are classifiable and therefore in the class of [EGL1]. For simple AH algebras of real rank zero, the corresponding decomposition property is called TAF by [Lin1], which is partially inspired by Popa's paper [Popa]. As proved by Lin ([Lin1-2]), a simple separable nuclear $\mathrm{C}^{*}$-algebra $A$ with UCT property is a TAI (or TAF, respectively) algebra if and only if $A$ is a simple AH algebra (or simple AH algebra of real rank zero) with no dimension growth, which is classified in [EGL1].

As in [EG2], let $T_{I I, k}$ be the 2-dimensional connected simplicial complex with $H_{1}\left(T_{I I, k}\right)=0$ and $H_{2}\left(T_{I I, k}\right)=$ $\mathbb{Z} / k \mathbb{Z}$, and let $I_{k}$ be the subalgebra of $M_{k}(C[0,1])=C\left([0,1], M_{k}(\mathbb{C})\right)$ consisting of all functions $f$ with the properties $f(0) \in \mathbb{C} 1_{k}$ and $f(1) \in \mathbb{C} \mathbf{1}_{k}$ (this algebra is called an Elliott dimension drop interval algebra). Denote $\mathcal{H D}$ the class of algebras consisting of direct sums of building blocks of the forms $M_{l}\left(I_{k}\right)$ and $P M_{n}(C(X)) P$, with $X$ being one of the spaces $\{p t\},[0,1], S^{1}$, and $T_{I I, k}$, and with $P \in M_{n}(C(X))$ being a projection. (In [DG], this class is denoted by $S H(2)$, and in [Jiang1], this class is denoted by $\mathcal{B}$ ). A $\mathrm{C}^{*}$-algebra is called an $\mathcal{A H D}$ algebra, if it is an inductive limit of algebras in $\mathcal{H D}$. In [GJL2], the authors classified all $\mathcal{A H D}$ algebras with the ideal property.

In [Jiang1], Jiang classified ATAI algebras $A$ under the extra restriction that $K_{1}(A)=\operatorname{tor} K_{1}(A)$. In this classification, Jiang used the scaled ordered total $K$-group (from [DG]) and the tracial state spaces $T(p A p)$ 
of cut-down algebras $p A p$, with certain compatibility conditions (from [Stev] and [Ji-Jiang]) as the invariantwe will call it $\operatorname{Inv^{0}}(A)$. In this paper, we will use the invariant $\operatorname{Inv^{0}}(A)$ together with the Hausdorffized algebraic $K_{1}$-group to deal with the torsion-free part of $K_{1}(A)$, with certain compatibility conditions-we will call this $\operatorname{Inv}(A)$. We will prove that this invariant reduces to Jiang's invariant in the case that $K_{1}(A)$ is a torsion group, that is, we removed the extra restriction that $K_{1}(A)=\operatorname{tor} K_{1}(A)$ of Jiang's classification in [Jiang1] and prove our theorem by reduced to the classification of $\mathcal{A H D}$ algebra with ideal property, which is done in [GJL1].

\section{Preliminaries And Definitions}

2.1. Let $A$ be a $\mathrm{C}^{*}$-algebra. Two projections in $A$ are said to be equivalent if they are Murray-von Neumann equivalent. We write $p \preceq q$ if $p$ is equivalent to a projection in $q A q$. We denote by $[p]$ the equivalent class of projections equivalent to $p$. Let $a \in A_{+}$, we write $p \preceq a$ if $p \preceq q$ for some projection $q \in a A a$.

Definition 2.2. Let $G \subset A$ be a finite set and $\delta>0$. We shall say that $\phi \in \operatorname{Map}(A, B)$ is $G-\delta$ multiplicative if $\|\phi(a b)-\phi(a) \phi(b)\|<\delta$ for all $a, b \in G$.

We also use $\operatorname{Map}_{G-\delta}(A, B)$ to denote all $G-\delta$ multiplicative maps.

If two maps $\varphi, \phi \in \operatorname{Map}(A, B)$ satisfy the condition $\|\phi(a)-\psi(a)\|<\delta$ for all $a \in G$, then we will write $\phi \approx_{\delta} \varphi$ on $G$.

If $G, H \subset A$ are two subsets of a $C^{*}$-algebra $A$ and for any $g \in G$, there is a $b \in H$ with $\|g-b\|<\delta$, then we denote $G \subset_{\delta} H$.

Definition 2.3. [Jiang1] We denote by $\mathcal{I}$ the class of all unital $\mathrm{C}^{*}$-algebras with the form $\bigoplus_{i=1}^{n} B_{i}$, where each $B_{i} \cong M_{k_{i}}$ or $B_{i} \cong M_{k_{i}}(C[0,1])$ for some $k_{(i)}$. Let $A \in \mathcal{I}$, we have the following:

(1) Every $\mathrm{C}^{*}$-algebra in $\mathcal{I}$ is of stable rank one.

(2) Two projections $p$ and $q$ in a $\mathrm{C}^{*}$-algebra $A \in \mathcal{I}$ are equivalent if and only if $\tau(p)=\tau(q)$ for all $\tau \in T(A)$, where $T(A)$ denotes the space of all tracial states.

(3) For any $\varepsilon>0$ and any finite subset $F \subset A$, there exist a number $\delta>0$ and a finite subset $G \subset A$ satisfying the following: If $L: A \rightarrow B$ is a $G-\delta$ multiplicative contractive completely positive linear map, where $B$ is a $\mathrm{C}^{*}$-algebra, then there exists a homomorphism $h: A \rightarrow B$ such that

$$
\|h(a)-L(a)\| \leq \varepsilon, \quad \forall a \in F
$$

Definition 2.4. ([Lin1]) A unital simple $\mathrm{C}^{*}$-algebra in $A$ is said to be tracially AI (TAI) if for any finite subset $F \subset A$ containing a nonzero element $b, \varepsilon>0$, integer $n>0$ and any full element $a \in A_{+}$, there exist nonzero projection $p \in A$ and a $\mathrm{C}^{*}$-algebra $I \subset A$ with $I \in \mathcal{I}$ and $1_{I}=p$ such that:

(1) $\|x p-p x\|<\varepsilon$ for all $x \in F$;

(2) $p x p \in_{\varepsilon} I$ for all $x \in F$;

(3) $n[1-p] \preceq[p]$ and $1-p \preceq a$. 
Definition 2.5. [Jiang1] A $C^{*}$-algebra $A$ (not necessary unital) is said to be ATAI algebra (approximately TAI algebra) if it is the inductive limit of a sequence of direct sums of simple unital TAI algebras with UCT.

2.6. Let $A$ and $B$ be two $C^{*}$-algebras. We use $\operatorname{Map}(A, B)$ to denote the space of all completely positive $*$ contractions from $A$ to $B$. If both $A$ and $B$ are unital, then $\operatorname{Map}(A, B)_{1}$ will denote the subset of $\operatorname{Map}(A, B)$ consisting of all such unital maps.

2.7. In the notation for an inductive limit system $\lim \left(A_{n}, \phi_{n, m}\right)$, we understand that

$$
\phi_{n, m}=\phi_{m-1, m} \circ \phi_{m-2, m-1} \circ \cdots \circ \phi_{n, n+1}
$$

where all $\phi_{n, m}: A_{n} \rightarrow A_{m}$ are homomorphisms.

We shall assume that, for any summand $A_{n}^{i}$ in the direct sum $A_{n}=\bigoplus_{i=1}^{t_{n}} A_{n}^{i}$, necessarily, $\phi_{n, n+1}\left(\mathbf{1}_{A_{n}^{i}}\right) \neq 0$, since, otherwise, we could simply delete $A_{n}^{i}$ from $A_{n}$, without changing the limit algebra.

If $A_{n}=\bigoplus_{i} A_{n}^{i}, A_{m}=\bigoplus_{j} A_{m}^{j}$, we use $\phi_{n, m}^{i, j}$ to denote the partial map of $\phi_{n, m}$ from the i-th block $A_{n}^{i}$ of $A_{n}$ to the j-th block $A_{m}^{j}$ of $A_{m}$. Also, we use $\phi_{n, m}^{-, j}$ to denote the partial map of $\phi_{n, m}$ from $A_{n}$ to $A_{m}^{j}$. That is, $\phi_{n, m}^{-, j}=\bigoplus \phi_{n, m}^{i, j}=\pi_{j} \phi_{n, m}$, where $\pi_{j}: A_{m} \rightarrow A_{m}^{j}$ is the canonical projection. We also use $\phi_{n, m}^{i,-}$ to denote $\left.\phi_{n, m}\right|_{A_{n}^{i}}: A_{n}^{i} \rightarrow A_{m}$.

2.8. An AH algebra is a $\mathrm{C}^{*}$-algebra which is $\lim \left(A_{n}=\bigoplus_{i=1}^{k_{n}} p_{n, i} M_{[n, i]}\left(C\left(X_{n, i}\right)\right) p_{n, i}, \phi_{n, m}\right)$, where each $X_{n, i}$ is a compact metrizable space, and $p_{n, i} \in M_{[n, i]}\left(C\left(X_{n, i}\right)\right)$ is a projection. Recall in [G1], Gong proved that a simple AH algebra with uniformly bounded dimension of local spectra, i.e. $\sup _{n, i} \operatorname{dim}\left(X_{n, i}\right)<\infty$ can be rewritten as $\mathrm{AH}$ inductive limit with spaces being $[0,1], S^{1}, S^{2}, T_{I I, k}, T_{I I I, k}$, where each $T_{I I, k}$ (or $T_{I I I, k}$ respectively) is two-dimensional (or three-dimensional respectively) connected simplicial complexes with $H^{2}\left(T_{I I, k}\right)=\mathbb{Z} / k \mathbb{Z}$ and $H^{1}\left(T_{I I, k}\right)=0$ (or with $H^{3}\left(T_{I I I, k}\right)=\mathbb{Z} / k \mathbb{Z}$ and $H^{1}\left(T_{I I I, k}\right)=0, H^{2}\left(T_{I I I, k}\right)=0$ ).

For any positive $k$ the dimension drop interval algebra $I_{k}$ is defined as

$$
I_{k}=\left\{f \in M_{k}(C[0,1]) \mid f(0)=\lambda \mathbf{1}_{k}, f(1)=\mu \mathbf{1}_{k}, \lambda, \mu \in \mathbb{C}\right\} .
$$

2.9. [GJL1] Let $X$ be a compact space and $\psi: C(X) \rightarrow P M_{k_{1}}(C(Y)) P(\operatorname{rank}(P)=k)$ be a unital homomorphism. For any point $y \in Y$, there are $k$ mutually orthogonal rank-1 projections $p_{1}, p_{2}, \cdots, p_{k}$ with $\sum_{i=1}^{k} p_{i}=P(y)$ and $\left\{x_{1}(y), x_{2}(y), \cdots, x_{k}(y)\right\} \subset X$ (may be repeat) such that $\psi(f)(y)=\sum_{i=1}^{k} f\left(x_{i}(y)\right) p_{i}, \forall f \in$ $C(X)$. We denote the set $\left\{x_{1}(y), x_{2}(y), \cdots, x_{k}(y)\right\}$ (counting multiplicities), by $S p \psi_{y}$. We shall call $S p \psi_{y}$ the spectrum of $\psi$ at the point $y$.

For any $f \in I_{k}$, let function $\underline{f}:[0,1] \rightarrow \mathbb{C} \bigsqcup M_{k}(\mathbb{C})$ (disjoint union) be defined by

$$
\underline{f}(t)= \begin{cases}\lambda, & \text { if } t=0 \text { and } f(0)=\lambda \mathbf{1}_{k} \\ \mu, & \text { if } t=1 \text { and } f(1)=\mu \mathbf{1}_{k} \\ f(t), & \text { if } 0<t<1\end{cases}
$$


That is, $\underline{f}(t)$ is the value of irreducible representation of $f$ corresponding to the point $t$. Similarly, for $f \in M_{l}\left(I_{k}\right)$, we can define $\underline{f}:[0,1] \longrightarrow M_{l}(\mathbb{C}) \sqcup M_{l k}(\mathbb{C})$, by

$$
\underline{f}(t)= \begin{cases}a, & \text { if } t=0 \text { and } f(0)=a \otimes \mathbf{1}_{k} \\ b, & \text { if } t=1 \text { and } f(1)=b \otimes \mathbf{1}_{k} \\ f(t), & \text { if } 0<t<1\end{cases}
$$

Suppose that $\phi: I_{k} \rightarrow P M_{n}(C(Y)) P$ is a unital homomorphism. Let $r=\operatorname{rank}(P)$. For each $y \in Y$, there are $t_{1}, t_{2}, \cdots, t_{m} \in[0,1]$ and a unitary $u \in M_{n}(\mathbb{C})$ such that

$$
P(y)=u\left(\begin{array}{cc}
\mathbf{1}_{\operatorname{rank}(P)} & 0 \\
0 & 0
\end{array}\right) u^{*}
$$

and

$$
\phi(f)(y)=u\left(\begin{array}{ccccc}
\underline{f}\left(t_{1}\right) & & & & \\
& \underline{f}\left(t_{2}\right) & & & \\
& & \ddots & & \\
& & \underline{f}\left(t_{m}\right) & \\
& & & \mathbf{0}_{n-r}
\end{array}\right) u^{*} \in P(y) M_{n}(\mathbb{C}) P(y) \subset M_{n}(\mathbb{C})
$$

for all $f \in I_{k}$.

We define the set $S p \phi_{y}$ to be the points $t_{1}, t_{2}, \cdots, t_{m}$ with possible fraction multiplicity. If $t_{i}=0$ or 1 , For example if we assume

$$
t_{1}=t_{2}=t_{3}=0<t_{4} \leq t_{5} \leq \cdots \leq t_{m-2}<1=t_{m-1}=t_{m},
$$

then $S p \phi_{y}=\left\{0^{\sim \frac{1}{k}}, 0^{\sim \frac{1}{k}}, 0^{\sim \frac{1}{k}}, t_{4}, t_{5}, \cdots, t_{m-2}, 1^{\sim \frac{1}{k}}, 1^{\sim \frac{1}{k}}\right\}$, which can also be written as

$$
S p \phi_{y}=\left\{0^{\sim \frac{3}{k}}, t_{4}, t_{5}, \cdots, t_{m-2}, 1^{\sim \frac{2}{k}}\right\}
$$

Here we emphasize that, for $t \in(0,1)$, we do not allow the multiplicity of $t$ to be non-integral. Also for 0 or 1 , the multiplicity must be multiple of $\frac{1}{k}$ (other fraction numbers are not allowed).

Let $\psi: C[0,1] \rightarrow P M_{n}(C(Y)) P$ be defined by the following composition

$$
\psi: C[0,1] \hookrightarrow I_{k} \stackrel{\phi}{\longrightarrow} P M_{n}(C(Y)) P
$$

where the first map is the canonical inclusion. Then we have $S p \psi_{y}=\left\{S p \phi_{y}\right\}^{\sim k}$-that is, for each element $t \in(0,1)$, its multiplicity in $S p \psi_{y}$ is exactly $k$ times of the multiplicity in $\phi_{y}$.

Recall that for $A=M_{l}\left(I_{k}\right)$, every point $t \in(0,1)$ corresponds to an irreducible representation $\pi_{t}$, defined by $\pi_{t}(f)=f(t)$. The representations $\pi_{0}$ and $\pi_{1}$ defined by

$$
\pi_{0}=f(0) \quad \text { and } \quad \pi_{1}=f(1)
$$

are no longer irreducible. We use $\underline{0}$ and $\underline{1}$ to denote the corresponding points for the irreducible representations. That is,

$$
\pi_{\underline{0}}(f)=\underline{f}(0) \quad \text { and } \quad \pi_{\underline{1}}(f)=\underline{f}(1) .
$$


Or we can also write $\underline{f}(0) \triangleq f(\underline{0})$ and $\underline{f}(1) \triangleq f(\underline{1})$. Then the equation $(*)$ could be written as

$$
\phi(f)(y)=u\left(\begin{array}{ccccc}
f\left(t_{1}\right) & & & & \\
& f\left(t_{2}\right) & & & \\
& & \ddots & & \\
& & & f\left(t_{m}\right) & \\
& & & & \mathbf{0}_{n-r}
\end{array}\right) u^{*}
$$

where some of $t_{i}$ may be $\underline{0}$ or $\underline{1}$. In this notation, $f(0)$ is equal to $\operatorname{diag}(\underbrace{f(\underline{0}), f(\underline{0}), \cdots, f(\underline{0})}_{k})$ up to unitary equivalence.

Under this notation, we can also write $0^{\sim \frac{1}{k}}$ as $\underline{0}$. Then the example of $S p \phi_{y}$ can be written as

$$
S p \phi_{y}=\left\{0^{\sim \frac{1}{k}}, 0^{\sim \frac{1}{k}}, 0^{\sim \frac{1}{k}}, t_{4}, t_{5}, \cdots, t_{m-2}, 1^{\sim \frac{1}{k}}, 1^{\sim \frac{1}{k}}\right\}=\left\{\underline{0}, \underline{0}, \underline{0}, t_{4}, t_{5}, \cdots, t_{m-2}, \underline{1}, \underline{1}\right\}
$$

2.10. We use $\mathcal{H D}$ to denote all $\mathrm{C}^{*}$-algebras $C=\bigoplus C^{i}$, where each $C^{i}$ is of the forms $M_{l}\left(I_{k}\right)$ or $P M_{n} C(X) P$ with $X$ being one of the spaces $\{p t\},[0,1], S^{1}, T_{I I, k}$. Each block $C^{i}$ will be called a basic $\mathcal{H D}$ block or a basic building block.

By $\mathcal{A H D}$ algebra, we mean the inductive limit of

$$
A_{1} \stackrel{\phi_{1,2}}{\longrightarrow} A_{2} \stackrel{\phi_{2,3}}{\longrightarrow} \cdots \longrightarrow A_{n} \cdots \longrightarrow \cdots,
$$

where $A_{n} \in \mathcal{H D}$ for each $n$.

For an $\mathcal{A H D}$ inductive limit $A=\lim \left(A_{n}, \phi_{n, m}\right)$, we write $A_{n}=\bigoplus_{i=1}^{t_{n}} A_{n}^{i}$, where $A_{n}^{i}=P_{n, i} M_{[n, i]}\left(C\left(X_{n, i}\right)\right) P_{n, i}$ of $A_{n}^{i}=M_{[n, i]}\left(I_{k_{n, i}}\right)$. For convenience, even for a block $A_{n}^{i}=M_{[n, i]}\left(I_{k_{n, i}}\right)$, we still use $X_{n, i}$ for $S p\left(A_{n}^{i}\right)=$ $[0,1]$-that is, $A_{n}^{i}$ is regarded as a homogeneous algebra or a sub-homogeneous algebra over $X_{n, i}$.

Definition 2.11. ([EG2], [DG]) Let $X$ be a compact connected space and let $P \in M_{N}(C(X))$ be a projection of rank $n$. The weak variation of a finite set $F \subset P M_{N}(C(X)) P$ is defined by

$$
\omega(F)=\sup _{\pi, \sigma} \inf _{u \in U(n)} \max _{a \in F}\left\|u \pi(a) u^{*}-\sigma(a)\right\|,
$$

where $\pi, \sigma$ run through the set of irreducible representations of $P M_{N} C(X) P$ into $M_{n}(\mathbb{C})$.

For $F \subset M_{r}\left(I_{k}\right)$, we define $\omega(F)=\omega(\imath(F))$, where $\imath: M_{r}\left(I_{k}\right) \longrightarrow M_{r k}(C[0,1])$ is the canonical embedding and $\imath(F)$ is regarded as a finite subset of $M_{r k}(C[0,1])$. Let $A$ be a basic $\mathcal{H D}$ block, a finite set $F \subset A$ is said to be weakly approximately constant to within $\varepsilon$ if $\omega(F)<\varepsilon$.

2.12. Let $\underline{K}(A)=K_{*}(A) \oplus \bigoplus_{k=2}^{\infty} K_{*}(A, \mathbb{Z} / k)$ be as in [DG]. Let $\Lambda$ be the Bockstein operation between $\underline{K}(A) \mathrm{s}$ (see 4.1 of $[\mathrm{DG}])$. It is well known that

$$
K_{*}(A, \mathbb{Z} \oplus \mathbb{Z} / k) \cong K_{0}\left(A \otimes C\left(T_{I I, k} \times S^{1}\right)\right) .
$$

As in $[D G]$, let

$$
K_{*}(A, \mathbb{Z} \oplus \mathbb{Z} / k)^{+} \cong K_{0}\left(A \otimes C\left(T_{I I, k} \times S^{1}\right)\right)^{+} .
$$

and let $\underline{K}(A)^{+}$to be the semigroup of $\underline{K}(A)$ generated by $\left\{K_{*}(A, \mathbb{Z} \oplus \mathbb{Z} / k \mathbb{Z})^{+}, k=2,3, \cdots\right\}$. 
2.13. Let $\operatorname{Hom}_{\Lambda}(\underline{K}(A), \underline{K}(B))$ be the homomorphism between $\underline{K}(A)$ and $\underline{K}(B)$ compatible with Bockstein operation $\Lambda$. Associativity of Kasparov $K K$-product gives a map

$$
\Gamma: K K(A, B) \rightarrow \operatorname{Hom}_{\Lambda}(\underline{K}(A), \underline{K}(B))
$$

Recall that every element $\alpha \in K K(A, B)$ defines a map $\alpha_{*} \in H o m_{\Lambda}(\underline{K}(A), \underline{K}(B))$. That is, it gives a sequence of homomorphisms

$$
\alpha^{i}: K_{i}(A) \longrightarrow K_{i}(B) \text { for } i=0,1 \text {, and } \alpha_{k}^{i}: K_{i}(A, \mathbb{Z} / k) \longrightarrow K_{i}(B, \mathbb{Z} / k) \text { for } i=0,1,
$$

which are compatible with the Bockstein operation $\Lambda$.

2.14. Let $\underline{K}(A)_{+}$be defined in 4.6 of $[\mathrm{DG}]$. Notice that, from [DG, 4.7], $\underline{K}(A)^{+} \subset \underline{K}(A)_{+}$, where for any unital $\mathrm{C}^{*}$-algebra $B$, let

$K K(A, B)_{+}=\left\{\alpha \in K K(A, B), \alpha\left(\underline{K}(A)_{+}\right) \subseteq \underline{K}(B)_{+}\right\}, \quad K K(A, B)_{D,+}=\left\{\alpha \in K K(A, B)_{+}, \alpha\left[1_{A}\right] \leq\left[1_{A}\right]\right\}$.

2.15. Let $A=P M_{\bullet} C\left(T_{I I, k}\right) P$, where we use $\bullet$ to denote any possible positive integers. From 5.14 of [G3], we know that an element $\alpha \in K K(A, B)$ is completely determined by $\alpha^{0}: K_{0}\left(C\left(T_{I I, n}\right)\right) \longrightarrow K_{0}(B)$ and $\alpha_{k}^{1}: K_{1}\left(C\left(T_{I I, k}\right), \mathbb{Z} / k\right) \longrightarrow K_{1}(B, \mathbb{Z} / k), k=0,1,2, \cdots$.

For any positive integer $k \geq 2$, denote

$$
K_{(k)}(A)=K_{0}(A) \oplus K_{1}(A) \oplus K_{0}(A, \mathbb{Z} / k) \oplus K_{1}(A, \mathbb{Z} / k) \text { and } K_{(k)}(A)_{+}=\underline{K}(A)_{+} \cap K_{(k)}(A)
$$

Then for $A=P M_{\bullet} C\left(T_{I I, k}\right) P$, an element $\alpha \in K K(A, B)$ is in $K K(A, B)_{+}$if and only if $\alpha\left(K_{(k)}(A)_{+}\right) \subset$ $K_{(k)}(B)_{+}, k=0,1$. Note that $K_{(k)}(A)_{+}$is finitely generated.

2.16. Note that, for any $\mathrm{C}^{*}$-algebra $A$, it follows from Künneth Theorem that

$$
K_{0}\left(A \otimes C\left(W_{k} \times S^{1}\right)\right)=K_{0}(A) \oplus K_{1}(A) \oplus K_{0}(A, \mathbb{Z} / k) \oplus K_{1}(A, \mathbb{Z} / k)
$$

where $W_{k}=T_{I I, k}$.

One can choose finite set $\mathcal{P} \subset M_{\bullet}\left(A \otimes C\left(W_{k} \times S^{1}\right)\right)$ of projections such that

$$
\left\{[P] \in K_{0}(A) \oplus K_{1}(A) \oplus K_{0}(A, \mathbb{Z} / k) \oplus K_{1}(A, \mathbb{Z} / k) \mid P \in \mathcal{P}(A)\right\} \subset \underline{K}(A)
$$

generate $K_{(k)}(A)=K_{0}(A) \oplus K_{1}(A) \oplus K_{0}(A, \mathbb{Z} / k) \oplus K_{1}(A, \mathbb{Z} / k)$, where $W_{k}=T_{I I, k}$.

If we choose finite set $G(\mathcal{P}) \subset A$ large enough and $\delta(\mathcal{P})>0$ small enough, then every $G(\mathcal{P})-\delta(\mathcal{P})$ multiplicative contraction $\phi: A \rightarrow B$ determines a map $\phi_{*}: \mathcal{P} \underline{K}(A) \rightarrow \underline{K}(B)$ which is compatible with the Bockstein operation $\Lambda$ (see [GL]). If $A=P M_{\bullet} C\left(T_{I I, k}\right) P$, then it also defines a $K K$ element $[\phi] \in K K(A, B)$.

Let $A=P M_{\bullet} C\left(T_{I I, k}\right) P$, recall that for $G \supseteq G(\mathcal{P}), \delta \leq \delta(\mathcal{P})$, a $G-\delta$ multiplicative map $\phi: A \rightarrow B$ is called a quasi $\mathcal{P} \underline{K}$ homomorphism, if there is a homomorphism $\psi: A \rightarrow B$ satisfying $\phi_{*}=\psi_{*}: \mathcal{P} \underline{K}(A) \rightarrow$ $\underline{K}(B)$. If $\phi$ is a quasi $\mathcal{P} \underline{K}$ homomorphism, then $[\phi] \in K K(A, B)_{+}$.

Suppose that $A=P M_{\bullet} C\left(T_{I I, k}\right) P$ and suppose that $B=\bigoplus_{i=1}^{n} B^{i}$ is a direct sum of basic $\mathcal{H D}$ bulding blocks. Let $\alpha \in K K(A, B)$, then $\alpha \in K K(A, B)_{+}$if and only if for each $i \in\{1,2, \cdots, n\}, \alpha^{i} \in K K\left(A, B^{i}\right)$ is either 
zero or $\alpha^{i}\left(\mathbf{1}_{C\left(T_{I I, k}\right)}\right)>0$ in $K_{0}\left(B^{i}\right)$. Recall that in [DG], a $K K$ element $\alpha \in K K\left(A, B=\bigoplus_{i=1}^{n} B^{i}\right)$ is called m-large if $\operatorname{rank}\left(\alpha^{i}\left(\mathbf{1}_{A}\right)\right) \geq m \cdot \operatorname{rank}\left(\mathbf{1}_{A}\right)$, for all $i \in\{1,2, \cdots, n\}$. By 5.5 and 5.6 of [DG] if $\alpha \in K K(A, B)_{+}$ is 6-large and $\alpha\left(\left[\mathbf{1}_{A}\right]\right) \leq\left[\mathbf{1}_{B}\right]$, then $\alpha$ can be realized by a homomorphism from $A$ to $B$.

2.17. Recall that the scale of $\mathrm{A}$, denoted by $\sum A$, is a subset of $K_{0}(A)$ consisting of $[p]$, where $p \in A$ is a projection. As all the $\mathrm{C}^{*}$-algebras $A$ in this paper have cancellation of projections, if $A$ has unit $\mathbf{1}_{A}$, then

$$
\sum A=\left\{x \in K_{0}(A) ; 0 \leq x \leq\left[\mathbf{1}_{A}\right]\right\} .
$$

For two $\mathrm{C}^{*}$-algebras $A, B$, by a "homomorphism" $\alpha$ from $\left(\underline{K}(A), \underline{K}(A)^{+}, \sum A\right)$ to $\left(\underline{K}(B), \underline{K}(B)^{+}, \sum B\right)$, it means a system of maps

$$
\alpha_{k}^{i}: K_{i}(A, \mathbb{Z} / k) \rightarrow K_{i}(B, \mathbb{Z} / k) ; \quad i=0,1, \quad k=0,2,3,4, \cdots
$$

which are compatible with Bockstein operation and $\alpha=\bigoplus_{k, i} \alpha_{k}^{i}$ satisfies $\alpha\left(\underline{K}(A)^{+}\right) \subseteq \underline{K}(B)^{+}$and finally $\alpha_{0}^{0}\left(\sum A\right) \subseteq \sum B$

2.18. For a unital $\mathrm{C}^{*}$-algebra $A$, let $T A$ denote the space of tracial states of $A$, i.e. $\tau \in T A$, if and only if $\tau$ is a positive linear map from $A$ to the complex plane $\mathbb{C}$, with $\tau(x y)=\tau(y x)$ and $\tau\left(\mathbf{1}_{A}\right)=1$. AffTA is the Banach space of all the continuous affine maps from $T A$ to $\mathbb{C}$. (In most references, AffT $A$ is defined to be the set of all the affine maps from $T A$ to $\mathbb{R}$. Our $A f f T A$ is a complexification of the standard $A f f T A$.) The element $\mathbf{1} \in \operatorname{Aff} T A$, defined by $\mathbf{1}(\tau)=1$, for all $\tau \in T A$, is called the unit of AffTA. AffTA, together with the positive cone $A f f T A_{+}$and the unit element 1 forms a scaled ordered complex Banach space. (Notice that for any element $x \in A f f T A$, there are $x_{1}, x_{2}, x_{3}, x_{4} \in A f f T A_{+}$such that $x=x_{1}-x_{2}+i x_{3}-i x_{4}$.)

There is a natural homomorphism $\rho_{A}: K_{0}(A) \rightarrow \operatorname{AffTA}$ defined by $\rho_{A}([p])(\tau)=\sum_{i=1}^{n} \tau\left(p_{i i}\right)$ for $\tau \in T A$ and $[p] \in K_{0}(A)$ represented by projection $p=\left(p_{i, j}\right) \in M_{n}(A)$.

Any unital homomorphism $\phi: A \rightarrow B$ induces a continuous affine map $T \phi: T B \rightarrow T A$, It turns out that $T \phi$ induces a unital positive linear map

$$
\text { AffT } \phi: \text { AffTA } \rightarrow \text { AffTB. }
$$

If $\phi: A \rightarrow B$ is not unital, we still have the positive linear map

$$
\text { AffT } \phi: \text { AffTA } \rightarrow \text { AffTB. }
$$

but it will not preserve the unit $\mathbf{1}$, only has property $\operatorname{AffT} \phi\left(\mathbf{1}_{A f f T A}\right) \leq \mathbf{1}_{A f f T B}$.

2.19. If $\alpha:\left(\underline{K}(A), \underline{K}(A)^{+}, \sum A\right) \rightarrow\left(\underline{K}(B), \underline{K}(B)^{+}, \sum B\right)$ is a homomorphism as in 2.17 , then for each projection $p \in A$, there is a projection $q \in B$ such that $\alpha[p]=[q]$.

Notice that for all the $\mathrm{C}^{*}$-algebras $A$ considered in this paper, the following is true: if $p_{1}, p_{2}$ are projections and $\left[p_{1}\right]=\left[p_{2}\right]$ in $K_{0}(A)$, then there is $u \in A$ with $u p_{1} u^{*}=p_{2}$. Therefore both $T(p A p)$ and $T(q B q)$ depend only on the class $[p] \in K_{0}(A)$ and $[q] \in K_{0}(B)$. For the classes $[p] \in \sum A\left(\subset K_{0}(A)\right), T(p A p)$ is taken as part of the invariant $\operatorname{Inv} v^{0}(A)$. For two classes $[p] \in \sum A,[q] \in \sum B$, with $\alpha([p])=[q]$, we will consider the system of continuous affine maps $\xi_{p, q}: T(q B q) \rightarrow T(p A p)$. Such system of maps is said to be compatible if for any two projections $p_{1} \leq p_{2}$ with $\alpha\left(\left[p_{1}\right]\right)=\left[q_{1}\right], \alpha\left(\left[p_{2}\right]\right)=\left[q_{2}\right]$ and $q_{1} \leq q_{2}$, the following diagram is 
commutative:

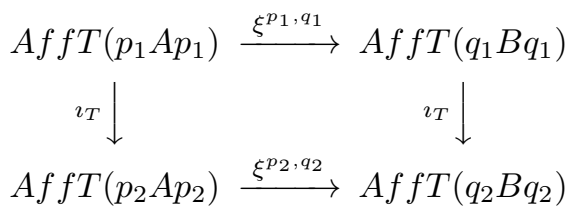

where the horizontal maps are induced by $\xi^{p_{1}, q_{1}}$ and $\xi^{p_{2}, q_{2}}$ respectively, and the vertical maps are induced by the inclusions $p_{1} A p_{1} \rightarrow p_{2} A p_{2}, q_{1} B q_{1} \rightarrow q_{2} B q_{2}$.

2.20. We denote $\left(\underline{K}(A), \underline{K}(A)^{+}, \sum A,\{T(p A p)\}_{[p] \in \sum A}\right)$ by $\operatorname{Inv} v^{0}(A)$. By a "map" between the invariants $\left(\underline{K}(A), \underline{K}(A)^{+}, \sum A,\{T(p A p)\}_{[p] \in \sum A}\right)$ and $\left(\underline{K}(B), \underline{K}(B)^{+}, \sum B,\{T(q B q)\}_{[q] \in \sum B}\right)$, we mean a map

$$
\alpha:\left(\underline{K}(A), \underline{K}(A)^{+}, \sum A\right) \rightarrow\left(\underline{K}(B), \underline{K}(B)^{+}, \sum B\right)
$$

as in 2.17 and maps $\xi^{p, q}: T(q B q) \rightarrow T(p A p)$ which are compatible as 2.19 . We denote this map by

$$
(\alpha, \xi):\left(\underline{K}(A), \underline{K}(A)^{+}, \sum A,\{T(p A p)\}_{[p] \in \sum A}\right) \rightarrow\left(\underline{K}(B), \underline{K}(B)^{+}, \sum B,\{T(q B q)\}_{[q] \in \sum B}\right)
$$

or simply

$$
(\alpha, \xi): \operatorname{Inv}^{0}(A) \rightarrow \operatorname{Inv}^{0}(B)
$$

2.21. Let $A$ be a unital $\mathrm{C}^{*}$-algebra. Let $U(A)$ denote the group of unitaries of $A$ and let $U_{0}(A)$ denote the connected component of $\mathbf{1}_{A}$ in $U(A)$. Let $D U(A)$ and $D U_{0}(A)$ denote the commutator subgroups of $U(A)$ and $U_{0}(A)$, respectively. (Recall that the commutator subgroup of a group $G$ is the subgroup generated by all elements of the form $a b a^{-1} b^{-1}$, where $a, b \in G$.) One introduces the following metric $D_{A}$ on $U(A) / \overline{D U(A)}$ (see $[\mathrm{NT}, \S 3])$. For $u, v \in U(A) / \overline{D U(A)}$

$$
D_{A}(u, v)=\inf \left\{\left\|u v^{*}-c\right\|: c \in \overline{D U(A)}\right\}
$$

where, on the right hand side of the equation, we use $u, v$ to denote any elements in $U(A)$, which represent the elements $u, v \in U(A) / \overline{D U(A)}$.

Denote the extended commutator group $D U^{+}(A)$, which is generated by $D U(A) \subset U(A)$ and the set $\left\{e^{2 \pi i t p}=e^{2 \pi i t} p+(\mathbf{1}-p) \in U(A) \mid t \in \mathbb{R}, p \in A\right.$ is a projection $\}$. Let $\widetilde{D U(A)}$ denote the closure of $D U^{+}(A)$. That is, $\widehat{D U(A)}=\overline{D U^{+}(A)}$

2.22. Let $A$ be a unital $C^{*}$-algebra. Let $A f f T A$ and $\rho_{A}: K_{0}(A) \rightarrow A f f T A$ be as defined as in 2.18 .

For simplicity, we will use $\rho K_{0}(A)$ to denote the set $\rho_{A}\left(K_{0}(A)\right)$. The metric $d_{A}$ on $A f f T A / \overline{\rho K_{0}(A)}$ is defined as follows (see [NT, §3]).

Let $d^{\prime}$ denote the quotient metric on $A f f T A \overline{\rho K_{0}(A)}$, i.e, for $f, g \in A f f T A \overline{\rho K_{0}(A)}$,

$$
d^{\prime}(f, g)=\inf \left\{\|f-g-h\|, h \in \overline{\rho K_{0}(A)}\right\} .
$$

Define $d_{A}$ by

$$
d_{A}(f, g)= \begin{cases}2, & \text { if } d^{\prime}(f, g) \geq \frac{1}{2} \\ \left|e^{2 \pi i d^{\prime}(f, g)}-1\right|, & \text { if } d^{\prime}(f, g)<\frac{1}{2}\end{cases}
$$

Obviously, $d_{A}(f, g) \leq 2 \pi d^{\prime}(f, g)$. 
Let $\rho \widetilde{K_{0}(A)}$ denote the closed real vector space spanned by $\overline{\rho K_{0}(A)}$. That is,

$$
\widetilde{\rho K_{0}(A)}:=\overline{\left\{\sum \lambda_{i} \phi_{i} \mid \lambda_{i} \in \mathbb{R}, \phi_{i} \in \rho K_{0}(A)\right\}} \text {. }
$$

For any $u, v \in U(A) / \widetilde{D U(A)}$, define

$$
\overline{D_{A}}(u, v)=\inf \left\{\left\|u v^{*}-c\right\|: c \in \widetilde{D U(A)}\right\} .
$$

Let $\vec{d}$ denote the quotient metric on $\operatorname{AffTA} / \widetilde{\rho K_{0}(A)}$, that is,

$$
\widetilde{d}(f, g)=\inf \left\{\|f-g-h\|, h \in \widetilde{\rho K_{0}(A)}\right\}, \quad f, g \in \operatorname{AffTA} / \widetilde{\rho K_{0}(A)} .
$$

Define $\widetilde{d}_{A}$ by

$$
\widetilde{d}_{A}(f, g)= \begin{cases}2, & \text { if } \vec{d}(f, g) \geq \frac{1}{2} \\ \left|e^{2 \pi i \vec{d}^{(}(f, g)}-1\right|, & \text { if } \vec{d}(f, g)<\frac{1}{2}\end{cases}
$$

2.23. Let

$$
\widetilde{S U(A)}:=\overline{\left\{x \in U(A) \mid x^{n} \in \widetilde{D U(A)} \text { for some } n \in \mathbb{Z}_{+} \backslash\{0\}\right\}} \text {. }
$$

and for simpler, denote $\widetilde{\rho K_{0}}(A) \triangleq \widetilde{\rho K_{0}(A)}, \widetilde{D U}(A)=\widetilde{D U(A)}, \widetilde{S U}(A)=\widetilde{S U(A)}$.

A unital homomorphism $\phi: A \rightarrow B$ induces a contractive group homomorphism

$$
\phi^{\natural}: U(A) / \widetilde{S U}(A) \longrightarrow U(B) / \widetilde{S U}(B) .
$$

If $\phi$ is not unital, then the map $\phi^{\natural}: U(A) / \widetilde{S U}(A) \longrightarrow U\left(\phi\left(\mathbf{1}_{A}\right) B \phi\left(\mathbf{1}_{A}\right)\right) / \widetilde{S U}\left(\phi\left(\mathbf{1}_{A}\right) B \phi\left(\mathbf{1}_{A}\right)\right)$ is induced by the corresponding unital homomorphism. In this case, $\phi$ also induces the map $\imath_{*} \circ \phi^{\natural}: U(A) / \widetilde{S U}(A) \longrightarrow$ $U(B) / \widetilde{S U}(B)$, which is denoted by $\phi_{*}$ to avoid confusion.

2.24. In [GJL1] and [GJL2], denote

$$
\left(\underline{K}(A), \underline{K}(A)^{+}, \sum A,\{\operatorname{AffT}(p A p)\}_{[p] \in \sum A},\{U(p A p) / \widetilde{S U}(p A p)\}_{[p] \in \sum A}\right)
$$

by $\operatorname{Inv}(A)$.

By a map from $\operatorname{Inv}(A)$ to $\operatorname{Inv}(B)$, one means

$$
\alpha:\left(\underline{K}(A), \underline{K}(A)^{+}, \sum A\right) \longrightarrow\left(\underline{K}(B), \underline{K}(B)^{+}, \sum B\right)
$$

as in 2.17, and for each pair $([p],[\bar{p}]) \in \sum A \times \sum B$ with $\alpha([p])=[\bar{p}]$, there are an associate unital positive linear map

$$
\xi^{p, \bar{p}}: \operatorname{AffT}(p A p) \longrightarrow \operatorname{AffT}(\bar{p} B \bar{p})
$$

and an associate contractive group homomorphism

$$
\chi^{p, \bar{p}}: U(p A p) / \widetilde{S U}(p A p) \longrightarrow U(\bar{p} B \bar{p}) / \widetilde{S U}(\bar{p} B \bar{p})
$$


satisfying the following compatibility conditions:

(a) If $p<q$, then the diagrams

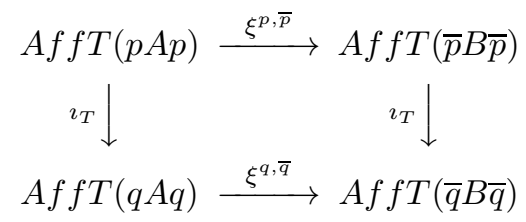

and

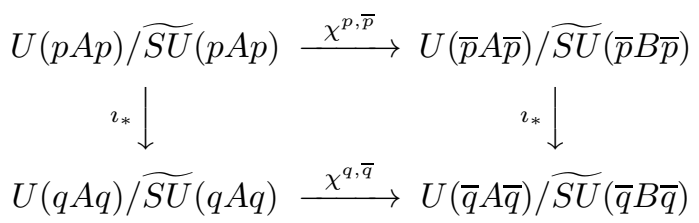

commutes, where the vertical maps are induced by inclusions.

(b) The following diagram commutes

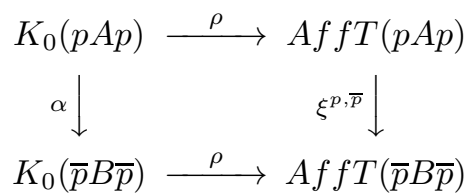

and therefore $\xi^{p, \bar{p}}$ induces a map (still denoted by $\xi^{p, \bar{p}}$ ):

$$
\xi^{p, \bar{p}}: A f f T(p A p) / \widetilde{\rho K_{0}}(p A p) \longrightarrow A f f T(\bar{p} B \bar{p}) / \widetilde{\rho K_{0}}(\bar{p} B \bar{p})
$$

(The commutativity of (III) follows from the commutativity of (I), by 1.20 of [Ji-Jiang]. So this is not an extra requirement.)

(c) The following diagrams

$$
\begin{aligned}
& A f f T(p A p) / \widetilde{\rho K_{0}}(p A p) \longrightarrow U(p A p) / \widetilde{S U}(p A p) \\
& \xi^{p, \bar{p}} \downarrow \chi^{p, \bar{p}} \downarrow \\
& A f f T(\bar{p} B \bar{p}) / \widetilde{\rho K_{0}}(\bar{p} B \bar{p}) \longrightarrow U(\bar{p} B \bar{p}) / \widetilde{S U}(\bar{p} B \bar{p})
\end{aligned}
$$

and

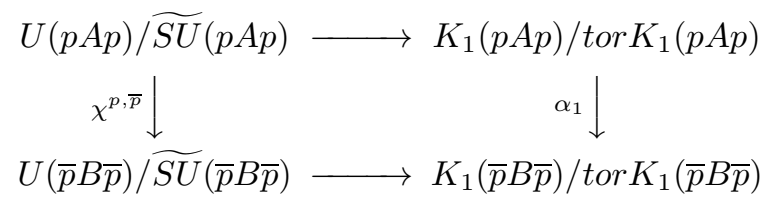

commute, where $\alpha_{1}$ is induced by $\alpha$.

We will denote the map from $\operatorname{Inv}(A)$ to $\operatorname{Inv}(B)$ by

$$
\begin{aligned}
&(\alpha, \xi, \chi):(\left(\underline{K}(A), \underline{K}(A)^{+}, \sum A,\{A f f T(p A p)\}_{[p] \in \sum A},\{U(p A p) / \widetilde{S U}(p A p)\}_{[p] \in \sum A}\right) \longrightarrow \\
&\left(\underline{K}(B), \underline{K}(B)^{+}, \sum B,\{A f f T(\bar{p} B \bar{p})\}_{[\bar{p}] \in \sum B},\{U(\bar{p} B \bar{p}) / \widetilde{S U}(\bar{p} B \bar{p})\}_{[\bar{p}] \in \sum B}\right)
\end{aligned}
$$

Note that $\operatorname{Inv} v^{0}$ is part of $\operatorname{Inv}(A)$. 


\section{Local approximation lemma}

Proposition 3.1. Let $A=P M_{\bullet} C\left(T_{I I, k}\right) P$ or $M_{l}\left(I_{k}\right)$ and $\mathcal{P}$ be as in 2.16. For any finite set $F \subset A, \varepsilon>0$, there exist a finite set $G \subset A(G \supset G(\mathcal{P})$ large enough), a positive number $\delta>0(\delta<\delta(\mathcal{P})$ small enough) such that the following statement is true:

If $B \in \mathcal{H D}, \phi, \psi \in \operatorname{Map}(A, B)$ are $G-\delta$ multiplicative and $\phi_{*}=\psi_{*}: \mathcal{P} \underline{K}(A) \rightarrow \underline{K}(B)$, then there is a homomorphism $\nu \in \operatorname{Hom}\left(A, M_{L}(B)\right)$ defined by point evaluations and there is a unitary $u \in M_{L+1}(B)$ such that $\left\|(\phi \oplus \nu)(a)-u(\psi \oplus \nu)(a) u^{*}\right\|<\varepsilon$ for all $a \in F$

Proof. For the special case that $A=P M_{\bullet} C\left(T_{I I, k}\right) P$ and $B$ is homogeneous, this is [G3, Theorem 5.18]. The proof of the general case is completely same (see 2.16 above). Note that, the calculation of $K_{0}\left(\Pi_{n=1}^{\infty} B_{n} / \bigoplus_{n=1}^{\infty} B_{n}\right)$, $K_{0}\left(\Pi_{n=1}^{\infty} B_{n} / \bigoplus_{n=1}^{\infty} B_{n}, \mathbb{Z} / k\right)$ and $K_{1}\left(\Pi_{n=1}^{\infty} B_{n} / \bigoplus_{n=1}^{\infty} B_{n}, \mathbb{Z} / k\right)$ in 5.12 of [G3] works well for the case that some of $B_{n}$ being of the form $M_{l}\left(I_{k}\right)$.

Lemma 3.2. Let $A=P M_{\bullet}(C(X)) P$ or $M_{l}\left(I_{k}\right)$. Let $F \subset A$ be approximately constant to within $\varepsilon$ (i.e., $\omega(F)<\varepsilon)$. Then for any two homomorphisms $\phi, \psi: A \rightarrow B$ defined by point evaluations with $K_{0} \phi=K_{0} \psi$, there exists a unitary $u \in B$ such that $\left\|\phi(f)-u \psi(f) u^{*}\right\|<2 \varepsilon \forall f \in F$.

Proof. The case $A=P M_{\bullet}(C(X)) P$ is Lemma 3.5 of [GJLP2]. For the case $A=M_{l}\left(I_{k}\right)$, one can also find a homomorphism $\phi^{\prime}: M_{l}(\mathbb{C}) \rightarrow B$ such that

$$
\left\|\phi(f)-\phi^{\prime}(f(\underline{0}))\right\|<\varepsilon \quad \forall f \in F,
$$

where $f(\underline{0})$ as in 2.9. Then one follows the same argument as the proof of Lemma 3.5 of [GJLP2] to get the result.

Lemma 3.3. Let $A=P M_{\bullet}(C(X)) P$ or $M_{l}\left(I_{k}\right), \varepsilon>0$, finite set $F \subset A$ with $\omega(A)<\varepsilon$. There exist a finite set $G \subset A(G \supset G(\mathcal{P}))$, a number $\delta>0(\delta<\delta(\mathcal{P}))$ and a positive integer $L$ such that the following statement is true. If $B \in \mathcal{H D}$, and $\phi, \psi \in \operatorname{Map}(A, B)_{1}$ are $G-\delta$ multiplicative and $\phi_{*}=\psi_{*}: \mathcal{P} \underline{K}(A) \rightarrow \underline{K}(B)$ and $\nu: A \rightarrow M_{\infty}(B)$ is a homomorphism defined by point evaluations with $\nu\left(\left[\mathbf{1}_{A}\right]\right) \geq L \cdot\left[\mathbf{1}_{B}\right] \in K_{0}(B)$, then there is a unitary $u \in\left(\mathbf{1}_{B} \oplus \nu\left(\mathbf{1}_{A}\right)\right) M_{\infty}(B)\left(\mathbf{1}_{B} \oplus \nu\left(\mathbf{1}_{A}\right)\right)$ such that

$$
\left\|(\phi \oplus \nu)(f)-u(\psi \oplus \nu)(f) u^{*}\right\|<5 \varepsilon \quad \forall f \in F .
$$

Proof. Let $L_{1}$ be as in Proposition 3.1 and $L=2 L_{1}$. Since $\nu\left(\left[\mathbf{1}_{A}\right]\right) \geq L \cdot\left[\mathbf{1}_{B}\right]=2 L_{1} \cdot\left[\mathbf{1}_{B}\right] \in K_{0}(B)$, there is a projection $Q<\nu\left(\mathbf{1}_{A}\right)$ such that $Q$ is equivalent to $\mathbf{1}_{M_{L_{1}}(B)}$. By Proposition 3.1, there exist a homomorphism $\nu_{1}: A \rightarrow Q M_{\infty}(B) Q$ defined by point evaluation and a unitary $w \in\left(\mathbf{1}_{B} \oplus \nu_{1}\left(\mathbf{1}_{A}\right)\right) M_{\infty}(B)\left(\mathbf{1}_{B} \oplus \nu_{1}\left(\mathbf{1}_{A}\right)\right)$ such that $\left\|\left(\phi \oplus \nu_{1}\right)(f)-w\left(\psi \oplus \nu_{1}\right)(f) w^{*}\right\|<\varepsilon \quad \forall f \in F$.

Since $\nu_{1}$ (and $\nu$ respectively) is homotopic to a homomorphism factoring through $M_{\operatorname{rank}(P)}(\mathbb{C})$ (for $A=$ $P M_{\bullet}(C(X)) P$ ) or factoring through $M_{l}(\mathbb{C})$ (for $A=M_{l}\left(I_{k}\right)$ ), there is a unital homomorphism $\nu_{2}: A \rightarrow$ $\left(\nu\left(\mathbf{1}_{A}\right)-\nu_{1}\left(\mathbf{1}_{A}\right)\right) M_{\infty}(B)\left(\nu\left(\mathbf{1}_{A}\right)-\nu_{1}\left(\mathbf{1}_{A}\right)\right)$ such that $K_{0}\left(\nu_{1} \oplus \nu_{2}\right)=K_{0}(\nu)$. 
By Lemma 3.2, $\nu$ is approximately unitarily equivalent to $\nu_{1} \oplus \nu_{2}$ to within $2 \varepsilon$ on $F$. Hence $\phi \oplus \nu$ is approximately unitarily equivalent to $\psi \oplus \nu$ on $F$ to within $2 \varepsilon+\varepsilon+2 \varepsilon=5 \varepsilon$.

Lemma 3.4. Let $A=P M_{\bullet} C\left(T_{I I, k}\right) P$, and $\mathcal{P} \subset M_{\bullet}\left(A \otimes C\left(W_{k} \times S^{1}\right)\right)$ as in 2.16. And let $B=\lim _{n \rightarrow \infty}\left(B_{n}, \psi_{n m}\right)$ be a unital simple inductive limit of direct sums of $\mathcal{H D}$ building blocks. Let $\phi: A \rightarrow B$ be a unital homomorphism. It follows that for any $G \supset G(\mathcal{P})$ and $\delta<\delta(\mathcal{P})$, there is a $B_{n}$ and a unital $G-\delta$ multiplicative contraction $\phi_{1}: A \rightarrow B_{n}$ which is a quasi $\mathcal{P} \underline{K}$-homomorphism such that $\left\|\psi_{n, \infty} \circ \phi_{1}(g)-\phi(g)\right\|<\delta$

Proof. There is a finite set $G_{1}$ and $\delta_{1}>0$ such that if a complete positive linear map $\psi: A \rightarrow C(C$ is a $\mathrm{C}^{*}$-algebra) and a homomorphism $\phi: A \rightarrow C$ satisfy that $\|\phi(g)-\psi(g)\|<\delta_{1}$ for all $g \in G_{1}$, then $\psi$ is $G-\frac{\delta}{2}$ multiplicative (see Lemma 4.40 in [G3]). Now if $\phi^{\prime}: A \rightarrow B_{l}$ satisfies $\left\|\psi_{l, \infty} \circ \phi^{\prime}(g)-\phi(g)\right\|<\delta$ for all $g \in G_{1}$, then $\psi_{l, \infty} \circ \phi^{\prime}$ is $G-\frac{\delta}{2}$ multiplicative. Hence for some $n>l$ (large enough), $\phi_{1}=\psi_{i, n} \circ \phi^{\prime}: A \rightarrow B_{n}$ is $G-\delta$ multiplicative. By replacing $G$ by $G \cup G_{1}$ and $\delta$ by $\min \left(\delta, \delta_{1}\right)$, we only need to construct a unital quasi $\mathcal{P} \underline{K}$-homomorsim $\phi_{1}: A \rightarrow B_{n}$ such that

$$
\left\|\psi_{n, \infty} \circ \phi_{1}(g)-\phi(g)\right\|<\delta, \quad \forall g \in G .
$$

Furthermore, if $\phi_{1}$ is a $G-\delta$ multiplicative map satisfying the above condition, then

$$
K K\left(\phi_{1}\right) \times K K\left(\psi_{n, \infty}\right)=K K(\phi) \in K K(A, B)_{+} \cdot
$$

Note that $K_{(k)}(A)_{+}$is finitely generated, there is an $m>n$ such that

$$
K K\left(\phi_{1}\right) \times K K\left(\psi_{n, m_{1}}\right) \in K K\left(A, B_{m_{1}}\right)_{+}
$$

Since $B$ is simple, for certain $m>m_{1}, K K\left(\psi_{n, m} \circ \phi_{1}\right)$ is 6-large and therefore can be realized by a homomorphism. That is, replacing $\phi_{1}$ by $\psi_{n, m} \circ \phi_{1}$, we get a quasi $\mathcal{P} \underline{K}$-homomorphism. Thus the proof of the lemma is reduced to the construction of $\phi_{1}$ to satisfy $(*)$.

Since $B=\lim _{n \rightarrow \infty}\left(B_{n}, \psi_{n m}\right)$, there is a $B_{n}$ and finite set $F \subset B_{n}$ such that $G \subset_{\frac{\delta}{3}} \psi_{n, \infty}(F)$. Since $B_{n}(\subset B)$ is a nuclear $\mathrm{C}^{*}$-algebra, there are two complete positive contractions $\lambda_{1}: B_{n} \rightarrow M_{N}(\mathbb{C})$ and $\lambda_{2}: M_{N}(\mathbb{C}) \rightarrow B_{n}$ such that

$$
\left\|\lambda_{2} \circ \lambda_{1}(f)-f\right\|<\frac{\delta}{3} \quad \forall f \in F .
$$

Since $B_{n}$ is a subalgebra of $B$, by Arversons Extension Theorem, one can extend the map $\lambda_{1}: B_{n} \rightarrow M_{N}(\mathbb{C})$ to $\beta_{1}: B \rightarrow M_{N}(\mathbb{C})$ such that $\beta_{1} \circ \psi_{n, \infty}=\lambda_{1}$.

One can verify that $\phi_{1}=\lambda_{2} \circ \beta_{1} \circ \phi: A \rightarrow B_{n}$ satisfies the condition $(*)$ as below. For $g \in G$, there is an $f \in F$ such that $\left\|\phi(g)-\psi_{n, \infty}(f)\right\|<\frac{\delta}{3}$, and therefore

$$
\begin{aligned}
&\left\|\psi_{n, \infty} \circ \phi_{1}(g)-\phi(g)\right\|=\left\|\left(\psi_{n, \infty} \circ \lambda_{2} \circ \beta_{1} \circ \phi_{1}\right)(g)-\phi(g)\right\| \\
& \leq\left\|\psi_{n, \infty}\left(\lambda_{2} \circ \beta_{1} \circ \phi_{1}(g)\right)-\psi_{n, \infty}\left(\lambda_{2} \circ \beta_{1} \circ \psi_{n, \infty}(f)\right)\right\| \\
&+\|\left(\psi_{n, \infty} \circ \lambda_{2} \circ \lambda_{1}(f)-\psi_{n, \infty}(f)\|+\|\left(\psi_{n, \infty}(f)-\phi(g) \|\right.\right. \\
& \leq \frac{\delta}{3}+\frac{\delta}{3}+\frac{\delta}{3}=\delta . \\
& 12
\end{aligned}
$$


This ends the proof of the lemma.

Lemma 3.5. For any finite set $F \subset P M_{\bullet} C\left(T_{I I, k}\right) P \triangleq B$ with $\omega(F)<\varepsilon$, there are a finite set $G \supset G(\mathcal{P})$, a positive number $\delta<\delta(\mathcal{P})$ and a positive integer $L$ (this $L$ will be denoted by $L(F, \varepsilon$ ) later), such that if $C$ is a $\mathcal{H D}$ basic building block, $p, q \in C$ are two projections with $p+q=\mathbf{1}_{C}$, and $\phi_{0}: B \rightarrow p C p$ and $\varphi_{1}: B \rightarrow q C q$ are two maps satisfying the following conditions:

(1) $\phi_{0}$ is quasi $\mathcal{P} \underline{K}$ homomorphism and $G-\delta$ multiplicative, and $\phi_{1}$ is defined by point evaluations (or equivalently, factoring through a finite dimensional $\mathrm{C}^{*}$-algebra), and

(2) $\operatorname{rank}(q) \geq L \operatorname{rank}(p)$,

then there is a homomorphism $\phi: B \rightarrow C$ such that $\left\|\phi_{0} \oplus \phi_{1}(f)-\phi(f)\right\|<5 \varepsilon$ for all $f \in F$.

Proof. Since $\phi_{0}$ is a quasi $\mathcal{P} \underline{K}$-homomorphism and is $G-\delta$ multiplicative, there is a homomorphism $\phi_{0}^{\prime}: B \rightarrow p C p$ such that $\phi_{0 *}^{\prime}=\phi_{0 *}: \mathcal{P} \underline{K}(B) \rightarrow \underline{K}(C)$. By Lemma 3.3, there is unitary $u \in C$ such that

$$
\left\|\left(\phi_{0} \oplus \phi_{1}\right)(f)-u\left(\phi_{0}^{\prime} \oplus \phi_{1}\right)(f) u^{*}\right\|<5 \varepsilon \quad \forall f \in F .
$$

The homomorphism $\phi=A d u^{*} \circ\left(\phi_{0}^{\prime} \oplus \phi_{1}\right)$ is as desired.

Lemma 3.6. Let $\varepsilon_{1}>\varepsilon_{2}>\cdots>\varepsilon_{n}>\cdots$ be a sequence with $\sum \varepsilon_{i}<+\infty$. Let $A$ be a simple $\mathrm{AH}$ algebra with no dimension growth (as in [EGL] and [Li4]). Then $A$ can be written as an $\mathcal{A H D}$ inductive limit

$$
A_{1}=\bigoplus_{i=1}^{t_{1}} A_{1}^{i} \longrightarrow A_{2}=\bigoplus_{i=1}^{t_{1}} A_{2}^{i} \longrightarrow \cdots
$$

with the unital subalgebras $B_{n}\left(=\bigoplus B_{n}^{i}\right) \subset A_{n}\left(=\bigoplus A_{n}^{i}\right.$ ) (that is, $\left.B_{n}^{i} \subset A_{n}^{i}\right)$ and $F_{n} \subset B_{n}$ and $G_{n} \subset A_{n}$ with $F_{n}=\bigoplus F_{n}^{i} \subset G_{n}=\bigoplus G_{n}^{i}$ such that the following statements hold:

(1) If $A_{n}^{i}$ is not of type $T_{I I}$, then $B_{n}^{i}=A_{n}^{i}$ and $F_{n}^{i}=F_{n}^{i}$. If $A_{n}^{i}$ is of type $T_{I I}$, then $B_{n}^{i}=P A_{n}^{i} P \oplus D_{n}^{i} \subset A_{n}^{i}$ with $F_{n}^{i}=\pi_{0}\left(F_{n}^{i}\right) \oplus \pi_{1}\left(F_{n}^{i}\right) \subset G_{n}^{i}$ and $\omega\left(\pi_{0}\left(F_{n}^{i}\right)\right)<\varepsilon$, where $D_{n}^{i}$ is a direct sum of the $\mathcal{H D}$ building blocks other than type $T_{I I}$, and $\pi_{0}: B_{n}^{i} \rightarrow P A_{n}^{i} P \triangleq B_{n}^{0, i}$ and $\pi_{1}: B_{n}^{i} \rightarrow D_{n}^{i}$ are canonical projections;

(2) $G_{n}^{i}$ generates $A_{n}^{i}, \phi_{n, n+1}\left(A_{n}\right) \subset B_{n+1}, \phi_{n, n+1}\left(G_{n}\right) \subset F_{n+1}$, and $\overline{\bigcup_{n=1}^{\infty} \phi_{n, \infty}\left(G_{n}\right)}=\overline{\bigcup_{n=1}^{\infty} \phi_{n, \infty}\left(F_{n}\right)}=$ unit ball of $A$;

(3) Suppose that both $A_{n}^{i}$ and $A_{n+1}^{j}$ are of type $T_{I I}$ and $\left.\phi \triangleq \pi_{0} \circ \phi_{n, n+1}^{i, j}\right|_{B_{n}^{0, i}}: B_{n}^{0, i} \rightarrow B_{n+1}^{0, j}$. Then $\phi\left(\mathbf{1}_{B_{n}^{0, i}}\right)=p_{0} \oplus p_{1} \in B_{n+1}^{0, j}$ and $\phi=\phi_{0} \oplus \phi_{1}$ with $\phi_{0} \in \operatorname{Hom}\left(B_{n}^{0, i}, p_{0} B_{n+1}^{0, j} p_{0}\right)_{1}, \phi_{1} \in \operatorname{Hom}\left(B_{n}^{0, i}, p_{1} B_{n+1}^{0, j} p_{1}\right)_{1}$ such that $\phi_{1}$ is defined by point evaluations (or equivalently, $\phi_{1}\left(B_{n}^{0, i}\right)$ is a finite dimensioned sub-algebra of $\left.p_{1} B_{n+1}^{0, j} p_{1}\right)$ and

$$
\left[p_{1}\right] \geq L\left(\pi_{0}\left(F_{n}^{i}\right), \varepsilon_{n}\right) \cdot\left[p_{0}\right]
$$

where $L\left(\pi_{0}\left(F_{n}^{i}\right), \varepsilon_{n}\right)$ is as in Lemma 3.5 (note that $\left.\omega\left(\pi_{0}\left(F_{n}^{i}\right)\right)<\varepsilon_{n}\right)$.

Proof. From [Li4], we know that $A$ can be written as an inductive limit of the direct sums of $\mathcal{H D}$ building blocks $A=\lim _{n \rightarrow \infty}\left(\widetilde{A_{n}}, \psi_{n m}\right)$. In our construction, we will choose $A_{n}=\widetilde{A_{k_{n}}}$ and homomorphisms $\phi_{n, n+1}: A_{n}=$ 
$\widetilde{A_{k_{n}}} \rightarrow A_{n+1}=\widetilde{A_{k_{n+1}}}$ satisfying $K K\left(\phi_{n, n+1}\right)=K K\left(\psi_{k_{n}, k_{n+1}}\right)$ and $\operatorname{AffT} \phi_{n, n+1}$ is close to AffT $\psi_{k_{n}, k_{n+1}}$ within any pregiven small number on a pregiven finite set, in such a way that $\phi_{n, n+1}$ also satisfies the desired condition as in the lemma with certain choices of subalgebras $B_{n} \subset A_{n}$ and and finite subsets $F_{n} \subset B_{n}$ and $G_{n} \subset A_{n}$.

Suppose that we already have $A_{n}=\widetilde{A_{k_{n}}}$ with the unital sub-algebra $B_{n}=\oplus_{i} B_{n}^{i}=\oplus_{i}\left(B_{n}^{0, i} \oplus D_{n}^{i}\right) \subset A_{n}=$ $\oplus_{i} A_{n}^{i}$, and two subsets $F_{n} \subset B_{n}, G_{n} \subset A_{n}$. as required in the lemma. We will construct, roughly, the next algebra $A_{n+1}=\widetilde{A_{k_{n+1}}}$, the sub-algebra $B_{n+1} \subset A_{n+1}$, two subsets $F_{n+1} \subset B_{n+1}, G_{n+1} \subset A_{n+1}$, and the homomorphism $\phi_{n, n+1}: A_{n} \rightarrow A_{n+1}$ to satisfy all the requirements in the lemma.

Applying the decomposition theorem-[G3, Theorem 4.37], as in the proof of the main theorem in [Li4], for $l>k_{n}$ large enough, and for each block $A_{n}^{i}=\widetilde{A_{k_{n}}^{i}}$ of type $T_{I I}$, the homomorphism $\psi_{k_{n}, l}^{i, j}$ can be decomposed into three parts $\phi_{0}^{1} \oplus \phi_{1} \oplus \phi_{2}$, roughly described as below: There are mutually orthogonal projections $Q_{0}, Q_{1}, Q_{2} \in \widetilde{A_{l}^{j}}$ with $\psi_{k_{n}, l}^{i, j}\left(\mathbf{1}_{A_{n}^{i}}\right)=Q_{0}+Q_{1}+Q_{2}$, there are two homomorphisms $\phi_{k} \in \operatorname{Hom}\left(A_{n}^{i}, Q_{k} \widetilde{A_{l}^{j}} Q_{k}\right)_{1}$, $(k=1,2)$ and a sufficient multiplicative quasi $\mathcal{P} \underline{K}$ homomorphism $\phi_{k} \in \operatorname{Map}\left(A_{n}^{i}, Q_{0} \widetilde{A_{l}^{j}} Q_{0}\right)_{1}$ possessing the following properties:

(i) $\phi_{0}^{1} \oplus \phi_{1} \oplus \phi_{2}$ is close to $\psi_{k_{n}, l}^{i, j}$ to within $\varepsilon_{n}$ on $G_{n}^{i}$;

(ii) $\phi_{0}^{1}\left(\mathbf{1}_{B_{n}^{0, i}}\right)$ is a projection and

$$
\left[Q_{1}\right] \geq L\left(\pi_{0}\left(F_{n}^{i}\right), \varepsilon\right) \cdot\left[Q_{0}\right] \quad \text { and } \quad \phi_{1}\left(\mathbf{1}_{B_{n}^{0, i}}\right) \geq L\left(\pi_{0}\left(F_{n}^{i}\right), \varepsilon\right) \cdot\left[\phi_{0}^{1}\left(\mathbf{1}_{B_{n}^{0, i}}\right)\right] ;
$$

(iii) $\phi_{1}$ is defined by point evaluation at a dense enough finite subset of $S p\left(\widetilde{A_{k_{n}}^{i}}\right)$ such that for any homomorphism $\phi_{0} \in \operatorname{Hom}\left(A_{n}^{i}, Q_{0} \widetilde{A_{l}^{j}} Q_{0}\right)_{1}$, we have $\omega\left(\phi_{0} \oplus \phi_{1}\left(G_{n}^{i}\right)\right)<\varepsilon_{n+1}$;

(iv) $\phi_{2}$ factors through an interval algebra $D_{1}^{i}$ as $\phi_{2}=\xi_{2}^{i} \circ \xi_{1}^{i}: A_{n}^{i} \stackrel{\xi_{1}^{i}}{\longrightarrow} D_{1}^{i} \stackrel{\xi_{2}^{i}}{\longrightarrow} \widetilde{A_{l}^{j}}$.

Then we can choose $k_{n+1}=l$ and $A_{n+1}=\widetilde{A_{k_{n+1}}}$. Note that $\phi_{0}^{1}$ is a quasi $\mathcal{P} \underline{K}$ homomorphism, one can choose $\phi_{0}: \widetilde{A_{k_{n}}^{i}} \rightarrow \phi_{0}^{1}\left(\mathbf{1}_{\overparen{A_{n}^{i}}}\right) \widetilde{A_{k_{n+1}}^{i}} \phi_{0}^{1}\left(\mathbf{1}_{\overparen{A_{k_{n}}^{i}}}\right)$ such that $K K\left(\phi_{0}^{1} \oplus \phi_{1} \oplus \phi_{2}\right)=K K\left(\phi_{0} \oplus \phi_{1} \oplus \phi_{2}\right)$ with $\phi_{0}^{1}\left(\mathbf{1}_{B_{n}^{0, i}}\right)=\phi_{0}\left(\mathbf{1}_{B_{n}^{0, i}}\right)$. Modify $\psi_{k_{n}, k_{n+1}}$ by replacing $\psi_{k_{n}, k_{n+1}}^{i, j}$ by $\phi_{0} \oplus \phi_{1} \oplus \phi_{2}$ to define $\phi_{n, n+1}: A_{n} \rightarrow A_{n+1}$.

Fix $j$ with $A_{n+1}^{j}=A_{k_{n+1}}^{j}$ being of type $T_{I I}$. Let $I_{0}=\left\{i \mid A_{n}^{i}=\widetilde{A_{k_{n}}^{j}}\right.$ is of type $\left.T_{I I}\right\}, I_{1}=\left\{i \mid A_{n}^{i}=\right.$ $\widetilde{A_{k_{n}}^{j}}$ is not of type $\left.T_{I I}\right\}$. Let $P_{i}=\left(\phi_{0} \oplus \phi_{1}\right)\left(\mathbf{1}_{A_{n}^{i}}\right) \in A_{n+1}^{j}$, let $P=\bigoplus_{i \in I_{0}} P_{i} \in A_{n+1}^{j}$ and $B_{n+1}^{0, j} \triangleq P A_{n+1}^{j} P$, and let $D_{n+1}^{j}=\bigoplus_{i \in I_{0}} \xi_{2}^{i}\left(D_{1}^{i}\right) \oplus \underset{i \in I_{1}}{\phi_{k_{n}, k_{n+1}}^{i, j}}\left(\widetilde{A_{k_{n}}^{j}}\right) \subset \widetilde{A_{k_{n+1}}^{j}}$ which is a direct sum of $\mathcal{H D}$ building blocks other than type $T_{I I}$. Let $B_{n+1}^{j}=B_{n+1}^{0, j} \oplus D_{n+1}^{j} \subset A_{n+1}^{j}$ if $A_{n+1}^{j}$ is of type $T_{I I}$; and $B_{n+1}^{j}=A_{n+1}^{j}$ if $A_{n+1}^{j}$ is not of type $T_{I I}$. Choose $G_{n+1}=\oplus G_{n+1}^{j}\left(\supset \phi_{n, n+1}\left(G_{n}\right)\right)$ sufficiently large. If $A_{n+1}^{j}$ is not of type $T_{I I}$, let $F_{n+1}^{j}=G_{n+1}^{j}$. If $A_{n+1}^{j}$ is of type $T_{I I}$, let $F_{n+1}^{j}=\phi_{n, n+1}^{-, j}\left(G_{n}\right)$.

Note that $K K\left(\psi_{k_{n}, k_{n+1}}\right)=K K\left(\phi_{n, n+1}\right)$. Since the rank of $\phi_{0}^{1}\left(\mathbf{1}_{\overparen{A_{k_{n}}^{i}}}\right)=\phi_{0}\left(\mathbf{1}_{\overparen{A_{k_{n}}^{i}}}\right)$ is much smaller than the ranks of $\phi_{1}\left(\mathbf{1}_{\overparen{A_{k}^{i, j}}}\right)$ and $\phi_{2}\left(\mathbf{1}_{\overparen{A_{k n}^{i, j}}}\right), \operatorname{AffT} \phi_{n, n+1}$ is very close to $\operatorname{AffT} \psi_{k_{n}, k_{n+1}}$. Hence $A^{\prime}=\lim \left(A_{n, n+1}, \phi_{n, m}\right)$ has the same Elliott invariant as $A=\lim \left(\widetilde{A_{k_{n}}}, \psi_{k_{n}, k_{m}}\right)$. Hence $A^{\prime} \cong A$. 
Corollary 3.7. Let $A$ be a simple $A H$ algebra with no dimension growth. And let $P_{1}, P_{2}, \cdots, P_{k} \in A$ be a set of mutually orthogonal projections. Then one can write A as inductive limit $A=\lim \left(A_{n}, \phi_{n, m}\right)$ with mutually orthogonal projections $P_{1}^{0}, P_{2}^{0}, \cdots, P_{k}^{0} \in A_{1}$ such that for each $i, \phi_{1, \infty}\left(P_{i}^{0}\right)=P_{i}$ and

$$
P_{i} A P_{i}=\lim _{n \rightarrow \infty}\left(\phi_{1, n}\left(P_{i}^{0}\right) A_{n} \phi_{1, n}\left(P_{i}^{0}\right),\left.\phi_{n, m}\right|_{\phi_{1, n}\left(P_{i}^{0}\right) A_{n} \phi_{1, n}\left(P_{i}^{0}\right)}\right)
$$

satisfies the properties of Lemma 3.6.

Proof. In the proof of Lemma 3.6, one can assume that there are $P_{1}^{\prime}, P_{2}^{\prime}, \cdots, P_{k}^{\prime} \in \widetilde{A_{1}}$ with $\psi_{1, \infty}\left(P_{i}^{\prime}\right)=P_{i}$ for all $i=1,2, \cdots, k$. Then the construction can be carried out to get our conclusion. Note that, applying Lemma 1.6.8 of [G3], one can strengthen [G3, Theorem 4.37] such that the following is true: For a set of pre-given orthogonal projections $p_{1}, p_{2}, \cdots, p_{k} \in A_{n}$, one can further require that $\psi_{0} \in \operatorname{Map}\left(A_{n}, Q_{0} A_{m} Q_{0}\right)$ satisfies that $\psi_{0}\left(p_{i}\right)$ are projections and $\left(\psi_{0} \oplus \psi_{1} \oplus \psi_{2}\right)\left(p_{i}\right)=\phi_{n, m}\left(p_{i}\right)$ for $i=1,2, \cdots, k$.

The following lemma is the main technique lemma of this section.

Lemma 3.8. Let $A, A^{\prime}$ be simple $\mathcal{A H D}$ inductive limit algebras with $A_{1} \stackrel{\phi_{1,2}}{\longrightarrow} A_{2} \stackrel{\phi_{2,3}}{\longrightarrow} \cdots \longrightarrow A_{n} \cdots \longrightarrow$ $A$ and $A_{1}^{\prime} \stackrel{\psi_{1,2}}{\longrightarrow} A_{2}^{\prime} \stackrel{\psi_{2,3}}{\longrightarrow} \cdots \longrightarrow A_{n}^{\prime} \cdots \longrightarrow A^{\prime}$ being described in Lemma 3.6. Let $\Lambda: A \rightarrow A^{\prime}$ be a homomorphism. Let $F \subset A_{m}$ be a finite set and $\varepsilon>0$. Then there is an $A_{l}^{\prime}$ and a homomorphism $\Lambda_{1}: A_{m} \rightarrow A_{l}^{\prime}$ such that

$$
\left\|\Lambda \circ \phi_{m, \infty}(f)-\psi_{l, \infty} \circ \Lambda_{1}(f)\right\|<\varepsilon \quad \forall f \in F .
$$

Proof. One can choose $n>m$ large enough such that $\phi_{m, n}(F) \subset_{\frac{\varepsilon}{4}} F_{n}$ and $5 \varepsilon_{n}<\frac{\varepsilon}{8}$. Note that $\phi_{m, n}\left(A_{m}\right) \subset$ $B_{n}$, where $B_{n}$ is as in Lemma 3.6. We will construct a homomorphism $\phi: B_{n} \rightarrow A_{l}^{\prime}$ such that

$$
\left\|\Lambda \circ \phi_{n, \infty}(f)-\psi_{l, \infty}(\phi(f))\right\|<\frac{\varepsilon}{2} \quad \forall f \in F_{n} \subset B_{n} \subset A_{n} .
$$

Then the homomorphism $\phi \circ \phi_{m, n}$ is as desired.

Let $I_{0}=\left\{i \mid B_{n}^{i}\right.$ is of type $\left.T_{I I}\right\}$ and $I_{1}=\left\{i \mid B_{n}^{i}\right.$ is not of type $\left.T_{I I}\right\}$. Then $\left\{\mathbf{1}_{B_{n}^{0, i}}\right\}_{i \in I_{0}} \cup\left\{\mathbf{1}_{D_{n}^{i}}\right\}_{i \in I_{0}} \cup\left\{\mathbf{1}_{B_{n}^{i}}\right\}_{i \in I_{1}}$ are mutually orthogonal projections. Hence $\left\{\Lambda \circ \phi_{n, \infty}\left(\mathbf{1}_{B_{n}^{0, i}}\right)\right\}_{i \in I_{0}} \cup\left\{\Lambda \circ \phi_{n, \infty}\left(\mathbf{1}_{D_{n}^{i}}\right)\right\}_{i \in I_{0}} \bigcup\left\{\Lambda \circ \phi_{n, \infty}\left(\mathbf{1}_{B_{n}^{i}}\right)\right\}_{i \in I_{1}}$ are mutually orthogonal projections in $A$. One can choose $n_{1}$ (large enough) and mutually orthogonal projections $\left\{P_{i}\right\}_{i \in I_{0}} \cup\left\{Q_{i}\right\}_{i \in I_{0}} \cup\left\{R_{i}\right\}_{i \in I_{1}} \subset A_{n_{1}}^{\prime}$ and a unitary $u \in A^{\prime}$ such that $\|u-1\|<\frac{\varepsilon}{16}$ and $\psi_{n_{1}, \infty}\left(P_{i}\right)=$ $u^{*}\left(\Lambda \circ \phi_{n, \infty}\left(\mathbf{1}_{B_{n}^{0, i}}\right)\right) u, \psi_{n_{1}, \infty}\left(Q_{i}\right)=u^{*}\left(\Lambda \circ \phi_{n, \infty}\left(\mathbf{1}_{D_{n}^{i}}\right)\right) u$ for $i \in I_{0}$ and $\psi_{n, \infty}\left(R_{i}\right)=u^{*}\left(\Lambda \circ \phi_{n, \infty}\left(\mathbf{1}_{B_{n}^{i}}\right)\right) u$ for $i \in I_{1}$.

Note that $\|A d u-i d\|<\frac{\varepsilon}{8}$. Replacing $\Lambda: A \rightarrow A^{\prime}$ by $\Lambda^{\prime}=A d u \circ \Lambda: A \stackrel{\Lambda}{\longrightarrow} A^{\prime} \stackrel{\text { Adu }}{\longrightarrow} A^{\prime}$ to make (*) true, it suffices to construct $\phi: B_{n} \rightarrow A_{l}^{\prime}$ for certain $l>n_{1}$ such that

$$
\left\|\Lambda^{\prime} \circ \phi_{n, \infty}(f)-\psi_{l, \infty} \circ \phi(f)\right\|<\frac{3 \varepsilon}{8} \quad \forall f \in F_{n} \subset B_{n} \subset A_{n}
$$

Such construction can be carried out for each of the blocks $B_{n}^{0, i}$ and $D_{n}^{i}$ for $i \in I_{0}, B_{n}^{i}$ for $i \in I_{1}$. Namely, we need to construct

$$
\left.\phi\right|_{B_{n}^{0, i}}: B_{n}^{0, i} \longrightarrow \psi_{n_{1}, l}\left(P_{i}\right) A_{l}^{\prime} \psi_{n_{1}, l}\left(P_{i}\right) \quad \forall i \in I_{0}
$$




$$
\begin{array}{cc}
\left.\phi\right|_{D_{n}^{i}}: D_{n}^{i} \longrightarrow \psi_{n_{1}, l}\left(Q_{i}\right) A_{l}^{\prime} \psi_{n_{1}, l}\left(Q_{i}\right) & \forall i \in I_{0} ; \\
\left.\phi\right|_{B_{n}^{i}}: B_{n}^{i} \longrightarrow \psi_{n_{1}, l}\left(R_{i}\right) A_{l}^{\prime} \psi_{n_{1}, l}\left(R_{i}\right) & \forall i \in I_{1} .
\end{array}
$$

separately, to satisfy the condition

$$
\left\|\Lambda^{\prime} \circ \phi_{n, \infty}(f)-\psi_{l, \infty} \circ \phi(f)\right\|<\frac{3 \varepsilon}{8}
$$

for all $f \in\left\{\pi_{0}\left(F_{n}^{i}\right)\right\}_{i \in I_{0}} \cup\left\{\pi_{1}\left(F_{n}^{i}\right)\right\}_{i \in I_{0}} \cup\left\{F_{n}^{i}\right\}_{i \in I_{1}}$.

For the blocks $\left\{D_{n}^{i}\right\}_{i \in I_{0}}$ and $\left\{B_{n}^{i}\right\}_{i \in I_{1}}$, the existence of such homomorphisms follows from the fact that the domain algebras are stably generated. So we only need to construct $\phi: B_{n}^{0, i} \longrightarrow \psi_{n_{1}, l}\left(P_{i}\right) A_{l}^{\prime} \psi_{n_{1}, l}\left(P_{i}\right)$ for $l$ large enough.

Let $J_{0}=\left\{j \mid B_{n+1}^{j}\right.$ is of type $\left.T_{I I}\right\}$ and $J_{1}=\left\{j \mid B_{n+1}^{i}\right.$ is not of type $\left.T_{I I}\right\}$. Let $\widetilde{P}^{i, j}=\pi_{0}\left(\phi_{n, n+1}^{i, j}\left(\mathbf{1}_{B_{n}^{0, i}}\right)\right) \in$ $B_{n+1}^{0, j}$ for $j \in J_{0}, \widetilde{Q}^{i, j}=\pi_{1}\left(\phi_{n, n+1}^{i, j}\left(\mathbf{1}_{B_{n}^{0, i}}\right)\right) \in D_{n+1}^{j}$ for $j \in J_{0}, \widetilde{R}^{i, j}=\pi_{1}\left(\phi_{n, n+1}^{i, j}\left(\mathbf{1}_{B_{n}^{0, i}}\right)\right) \in D_{n+1}^{j}$ for $j \in J_{1}$. (Here we only consider the case $i \in I_{0}$ ).

As in Lemma 3.6, we have the decomposition $\pi_{0} \circ \phi_{n, n+1}^{i, j}\left(\mathbf{1}_{B_{n}^{0, i}}\right)=p_{0}+p_{1} \in B_{n+1}^{0, j}$ and $\left.\pi_{0} \circ \phi_{n, n+1}^{i, j}\right|_{B_{n}^{0, i}}=$ $\phi_{0} \oplus \phi_{1}$ with $\phi_{0} \in \operatorname{Hom}\left(B_{n}^{0, i}, p_{0} B_{n+1}^{0, j} p_{0}\right)_{1}$ and $\phi_{1} \in \operatorname{Hom}\left(B_{n}^{0, i}, p_{1} B_{n+1}^{0, j} p_{1}\right)_{1}$. Denote $p_{0}, p_{1}$ by $p_{0}^{i, j}$ and $p_{1}^{i, j}$, then $\widetilde{P}^{i, j}=p_{0}^{i, j} \oplus p_{1}^{i, j}$. It follows that

$$
\left\{\Lambda^{\prime}\left(\phi_{n+1, \infty}\left(p_{0}^{i, j}\right)\right)\right\}_{j \in J_{0}} \bigcup\left\{\Lambda^{\prime}\left(\phi_{n+1, \infty}\left(p_{1}^{i, j}\right)\right)\right\}_{j \in J_{0}} \bigcup\left\{\Lambda^{\prime}\left(\phi_{n+1, \infty}\left(\widetilde{Q}^{i, j}\right)\right)\right\}_{j \in J_{0}} \bigcup\left\{\Lambda^{\prime}\left(\phi_{n+1, \infty}\left(\widetilde{R}^{i, j}\right)\right)\right\}_{j \in J_{1}}
$$

is a set of mutually orthogonal projections with sum to be $\Lambda^{\prime}\left(\phi_{n, \infty}\left(\mathbf{1}_{B_{n}^{0, i}}\right)\right)=\psi_{n_{1}, \infty}\left(P_{i}\right) \in A^{\prime}$. For $n_{2}>n_{1}$ (large enough), there are mutually orthogonal projections

$$
\left.\left\{P_{0}^{i, j}\right\}_{j \in J_{0}} \cup\left\{P_{1}^{i, j}\right)\right\}_{j \in J_{0}} \cup\left\{Q^{i, j}\right\}_{j \in J_{0}} \cup\left\{R^{i, j}\right\}_{j \in J_{1}} \subset \psi_{n_{1}, n_{2}}\left(P_{i}\right) A_{n_{2}}^{\prime} \psi_{n_{1}, n_{2}}\left(P_{i}\right)
$$

and a unitary $v \in \psi_{n, \infty}\left(P_{i}\right) A^{\prime} \psi_{n, \infty}\left(P_{i}\right)$ such that $\|v-1\|<\frac{\varepsilon}{16}$, and

$$
\begin{aligned}
& \psi_{n_{2}, \infty}\left(P_{0}^{i, j}\right)=v^{*}\left(\Lambda^{\prime}\left(\phi_{n+1, \infty}\left(p_{0}^{i, j}\right)\right)\right) v, \\
& \psi_{n_{2}, \infty}\left(P_{1}^{i, j}\right)=v^{*}\left(\Lambda^{\prime}\left(\phi_{n+1, \infty}\left(p_{1}^{i, j}\right)\right)\right) v, \\
& \psi_{n_{2}, \infty}\left(Q^{i, j}\right)=v^{*}\left(\Lambda^{\prime}\left(\phi_{n+1, \infty}\left(\widetilde{Q}^{i, j}\right)\right)\right) v
\end{aligned}
$$

for all $j \in J_{0}$; and

$$
\psi_{n_{2}, \infty}\left(R^{i, j}\right)=v^{*}\left(\Lambda^{\prime}\left(\phi_{n+1, \infty}\left(\widetilde{R}^{i, j}\right)\right)\right) v
$$

for all $j \in J_{1}$.

Let $\widetilde{\Lambda}=A d v \circ \Lambda^{\prime}$. Then the construction of $\left.\phi\right|_{B_{n}^{0, i}}$ satisfying $(* *)$ for all $f \in \pi_{0}\left(F_{0}^{i}\right)$ is reduced to the construction of homomorphisms

$$
\begin{gathered}
\xi_{0}^{j}: B_{n}^{0, j} \longrightarrow \psi_{n_{2}, l}\left(P_{0}^{i, j} \oplus P_{1}^{i, j}\right) A_{l}^{\prime} \psi_{n_{2}, l}\left(P_{0}^{i, j} \oplus P_{1}^{i, j}\right), \\
\xi_{1}^{j}: \widetilde{Q}^{i, j} D_{n+1}^{j} \widetilde{Q}^{i, j} \longrightarrow \psi_{n_{2}, l}\left(Q^{i, j}\right) A_{l}^{\prime} \psi_{n_{2}, l}\left(Q^{i, j}\right)
\end{gathered}
$$

for all $j \in J_{0}$; and

$$
\xi^{j}: \widetilde{R}^{i, j} B_{n+1}^{j} \widetilde{R}^{i, j} \longrightarrow \psi_{n_{2}, l}\left(R^{i, j}\right) A_{l}^{\prime} \psi_{n_{2}, l}\left(R^{i, j}\right)
$$


for all $j \in J_{1}$, such that

$$
\begin{gathered}
(* * *) \quad\left\|\widetilde{\Lambda} \circ \phi_{n, \infty}(f)-\psi_{l, \infty} \circ \xi_{0}^{j}(f)\right\|<\frac{\varepsilon}{4} \quad \forall f \in \pi_{0}\left(F_{n}^{i}\right) \subset B_{n}^{0, i} \quad \text { and } j \in J_{0} . \\
\left\|\widetilde{\Lambda} \circ \phi_{n+1, \infty}(f)-\psi_{l, \infty} \circ \xi_{1}^{j}(f)\right\|<\frac{\varepsilon}{4} \quad \forall f \in \pi_{1}\left(\left.\phi_{n, n+1}^{i, j}\right|_{B_{n}^{0, i}}\left(\pi_{0}\left(F_{n}^{i}\right)\right)\right) \subset \widetilde{Q}^{i, j} D_{n+1}^{j} \widetilde{Q}^{i, j} \quad \text { and } j \in J_{0} . \\
\left\|\widetilde{\Lambda} \circ \phi_{n+1, \infty}(f)-\psi_{l, \infty} \circ \xi^{j}(f)\right\|<\left.\frac{\varepsilon}{4} \quad \forall f \in \phi_{n, n+1}^{i, j}\right|_{B_{n}^{0, i}}\left(\pi_{0}\left(F_{n}^{i}\right)\right) \subset \widetilde{R}^{i, j} B_{n+1}^{j} \widetilde{R}^{i, j} \quad \text { and } j \in J_{1} .
\end{gathered}
$$

(Warning: The domain of $\xi_{0}^{j}$ is $B_{n}^{0, i}$ which is a sub-algebra of $B_{n}$ but not a sub-algebra of $B_{n+1}$. On the other hand, $\xi_{1}^{j}\left(j \in J_{0}\right)$ and $\xi^{j}\left(j \in J_{1}\right)$ are homomorphisms from $\widetilde{Q}^{i, j} D_{n+1}^{j} \widetilde{Q}^{i, j}\left(j \in J_{0}\right)$ and $\widetilde{R}^{i, j} B_{n+1}^{j} \widetilde{R}^{i, j}\left(j \in J_{1}\right)$ which are subalgebras of $\left.B_{n+1}\right)$.

The existence of the homomorphisms $\xi_{1}^{j}\left(j \in J_{0}\right)$ and $\xi^{j}\left(j \in J_{1}\right)$ follows from the fact that the corresponding domain algebras $\widetilde{Q}^{i, j} D_{n+1}^{j} \widetilde{Q}^{i, j}\left(j \in J_{0}\right)$ and $\widetilde{R}^{i, j} B_{n+1}^{j} \widetilde{R}^{i, j}\left(j \in J_{1}\right)$ are stably generatedof course, we need to choose $l>n_{2}$ large enough.

So we only need to construct $\xi_{0}^{j}$ to satisfy $(* * *)$ above. Let $G \supset G(\mathcal{P})$ and $\delta<\delta(\mathcal{P})$ be as in Lemma 3.5 for $\pi_{0}\left(F_{n}^{i}\right)$ and $\varepsilon_{n}$ (note that $\omega\left(\pi_{0}\left(F_{0}^{i}\right)\right)<\varepsilon_{n}$ ). Recall that $L\left(\pi_{0}\left(F_{n}^{i}\right), \varepsilon_{n}\right)$ is also from Lemma 3.5. Recall that $\phi_{0} \in \operatorname{Hom}\left(B_{n}^{0, i}, p_{0}^{i, j} B_{n+1}^{j} p_{0}^{i, j}\right), \phi_{1} \in \operatorname{Hom}\left(B_{n}^{0, i}, p_{1}^{i, j} B_{n+1}^{j} p_{1}^{i, j}\right)$, and that $\phi_{1}\left(B_{n}^{0, i}\right)$ is a finite dimensional algebra. There is a homomorphism $\lambda_{1}: \phi_{1}\left(B_{n}^{0, i}\right) \rightarrow \psi_{n_{2}, l}\left(P_{1}^{i, j}\right) A_{l}^{\prime} \psi_{n_{2}, l}\left(P_{1}^{i, j}\right)$ (for $l$ large enough) such that

$$
\left\|\psi_{l, \infty} \circ \lambda_{1}(f)-\widetilde{\Lambda}\left(\phi_{n+1, \infty}(f)\right)\right\|<\frac{\varepsilon}{16} \quad \forall f \in \phi_{1}\left(\pi_{0}\left(F_{n}^{i}\right)\right) .
$$

Applying Lemma 3.4 to the inductive $\operatorname{limit}, \lim \left(\psi_{n_{2}, m}\left(P_{0}^{i, j}\right) A_{m}^{\prime} \psi_{n_{2}, m}\left(P_{0}^{i, j}\right), \psi_{m, l}\right)$, the finite set $\phi_{0}\left(\pi_{0}\left(F_{n}^{i}\right)\right) \subset$ $p_{0}^{i, j} B_{n+1}^{0, j} p_{0}^{i, j}$ and the homomorphism $\widetilde{\Lambda} \circ \phi_{n+1, \infty}: p_{0}^{i, j} B_{n+1}^{0, j} p_{0}^{i, j} \rightarrow \psi_{n_{2}, \infty}\left(P_{0}^{i, j}\right) A_{m}^{\prime} \psi_{n_{2}, \infty}\left(P_{0}^{i, j}\right)$, for $l\left(>n_{2}\right)$ large enough, one can obtain a $\phi_{0}(G)-\delta$ multiplicative quasi- $\mathcal{P} \underline{K}$ homomorphism $\lambda_{0}: p_{0}^{i, j} B_{n+1}^{0, j} p_{0}^{i, j} \rightarrow$ $\psi_{n_{2}, l}\left(P_{0}^{i, j}\right) A_{m}^{\prime} \psi_{n_{2}, l}\left(P_{0}^{i, j}\right)$ such that

$$
\left\|\psi_{l, \infty} \circ \lambda_{0}(f)-\left(\widetilde{\Lambda} \circ \phi_{n+1, \infty}\right)(f)\right\|<\frac{\varepsilon}{16} \quad \forall f \in \phi_{0}\left(\pi_{0}\left(F_{n}^{i}\right)\right) .
$$

Let $\xi^{\prime}=\lambda_{0} \circ \phi_{0} \oplus \lambda_{1} \circ \phi_{1}: B_{n}^{0, i} \longrightarrow \psi_{n_{2}, l}\left(P_{0}^{i, j} \oplus P_{1}^{i, j}\right) A_{l}^{\prime} \psi_{n_{2}, l}\left(P_{0}^{i, j} \oplus P_{1}^{i, j}\right)$. Then

$$
(* * * *) \quad\left\|\widetilde{\Lambda} \circ \phi_{n, \infty}(f)-\psi_{l, \infty} \circ \xi^{\prime}(f)\right\|<\frac{\varepsilon}{8} \quad \forall f \in \pi_{0}\left(F_{n}^{i}\right) .
$$

On the other hand, since $\left[P_{1}^{i, j}\right] \geq L\left(\pi_{0}\left(F_{n}^{i}\right), \varepsilon_{n}\right) \cdot\left[P_{0}^{i, j}\right]$ in K-theory, and $\lambda_{1} \circ \phi_{1}$ is a homomorphism with finite dimensional image, we know that $\xi^{\prime}=\lambda_{0} \circ \phi_{0} \oplus \lambda_{1} \circ \phi_{1}$ satisfies the condition of Lemma 3.6-note that $\lambda_{0}$ is $\phi_{0}(G)-\delta$ multiplicative implies that $\lambda_{0} \circ \phi_{0}$ is $G-\delta$ multiplicative. By Lemma 3.6, there is a homomorphism $\xi_{0}^{j}: B_{n}^{0, j} \longrightarrow \psi_{n_{2}, l}\left(P_{0}^{i, j} \oplus P_{1}^{i, j}\right) A_{l}^{\prime} \psi_{n_{2}, l}\left(P_{0}^{i, j} \oplus P_{1}^{i, j}\right)$ such that $\left\|\xi_{0}^{j}(f)-\xi^{\prime}(f)\right\| \leq 5 \varepsilon_{n}<\frac{\varepsilon}{8}$. Combining with $(* * * *)$ we know that $\xi_{0}^{j}$ satisfies $(* * *)$ as desired, and therefore the lemma is proved.

Definition 3.9. A $\mathrm{C}^{*}$-algebra $A$ is said to have the ideal property if each closed two-sided ideal in $A$ is generated by its projections.

Remark 3.10. All simple, unital $\mathrm{C}^{*}$-algebras have the ideal property. The direct sum of TAI algebras have the ideal property. The inductive limit of $\mathrm{C}^{*}$-algebras with the ideal property have the ideal property. 
The following results is due to Gong, Jiang, Li.

Proposition 3.11. ([GJL1]) Suppose that $A=\lim \left(A_{n}, \phi_{n, m}\right)$ and $B=\lim \left(B_{n}, \psi_{n, m}\right)$ are two (not necessarily unital) $\mathcal{A H D}$ inductive limit algebras with the ideal property. Suppose that there is an isomorphism

$$
\alpha:\left(\underline{K}(A), \underline{K}(A)^{+}, \sum A\right) \longrightarrow\left(\underline{K}(B), \underline{K}(B)^{+}, \sum B\right)
$$

which is compatible with Bockstein operations. Suppose that for each projection $p \in A$ and $\bar{p} \in B$ with $\alpha([p])=[\bar{p}]$, there exist a unital positive linear isomorphism

$$
\xi^{p, \bar{p}}: A f f T(p A p) \longrightarrow A f f T(\bar{p} B \bar{p})
$$

and an isometric group isomorphism

$$
\gamma^{p, \bar{p}}: U(p A p) / \widetilde{S U}(p A p) \longrightarrow U(\bar{p} B \bar{p}) / \widetilde{S U}(\bar{p} B \bar{p})
$$

satisfying the following compatibility conditions:

(1) For each pair of projections $p<q<\in A$ and $\bar{p}<\bar{q}<\in B$ with $\alpha([p])=[\bar{p}], \alpha([q])=[\bar{q}]$, the diagrams

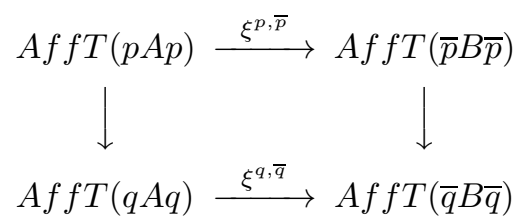

and

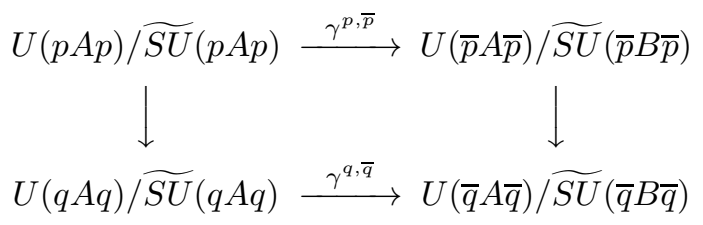

commute, where the vertical maps are induced by the inclusion homomorphisms.

(2) The maps $\alpha_{0}$ and $\xi^{p, \bar{p}}$ are compatible, that is, the diagram

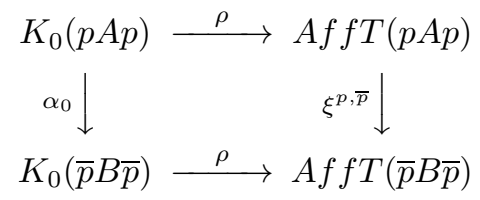

commutes (this is not an extra requirement, since it follows from the commutativity of the first diagram in (1) above by [Ji-Jiang]), and then we have the map (still denoted by $\xi^{p, \bar{p}}$ ):

$$
\xi^{p, \bar{p}}: \operatorname{AffT}(p A p) / \widetilde{\rho K_{0}}(p A p) \longrightarrow \operatorname{AffT}(\bar{p} B \bar{p}) / \widetilde{\rho K_{0}}(\bar{p} B \bar{p})
$$

(3) The map $\xi^{p, \bar{p}}$ and $\gamma^{p, \bar{p}}$ are compatible, that is, the diagram

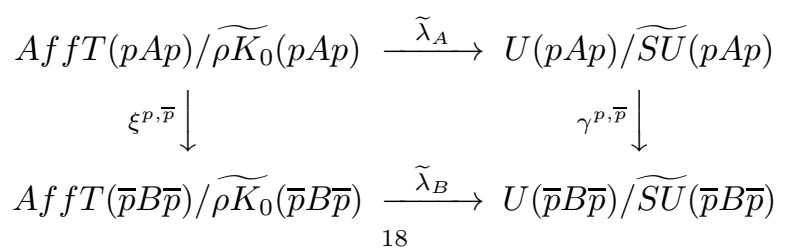


commutes.

(4) The map $\alpha_{1}: K_{1}(p A p) / \operatorname{tor} K_{1}(p A p) \longrightarrow K_{1}(\bar{p} B \bar{p}) / \operatorname{tor} K_{1}(\bar{p} B \bar{p})$ (note that $\alpha$ keeps the positive cone of $\underline{K}(A)^{+}$and therefore takes $K_{1}(p A p) \subset K_{1}(A)$ to $K_{1}(\bar{p} B \bar{p}) \subset K_{1}(B)$ is compatible with $\gamma^{p, \bar{p}}$, that is, the diagram

commutes.

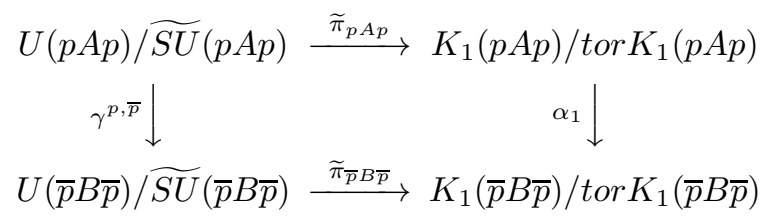

Then there is an isomorphism $\Gamma: A \rightarrow B$ such that

(a) $K(\Gamma)=\alpha$, and

(b)If $\Gamma_{p}: p A p \rightarrow \Gamma(p) B \Gamma(p)$ is the restriction of $\Gamma$, then $\operatorname{Aff} T\left(\Gamma_{p}\right)=\xi^{p, \bar{p}}$ and $\Gamma_{p}^{\natural}=\gamma^{p, \bar{p}}$, where $[\bar{p}]=[\Gamma(p)]$.

Proposition 3.10. ([GJL2, Proposition 2.38]) Let $A, B \in \mathcal{H D}$ or $\mathcal{A H D}$ be unital $\mathrm{C}^{*}$-algebras. Suppose that $K_{1}(A)=\operatorname{tor}\left(K_{1}(A)\right)$ and $K_{1}(B)=\operatorname{tor}\left(K_{1}(B)\right)$. It follows that $\operatorname{Inv}^{0}(A) \cong \operatorname{Inv}^{0}(B)$ implies that $\operatorname{Inv}(A) \cong \operatorname{Inv}(B)$.

\section{Main Theorems}

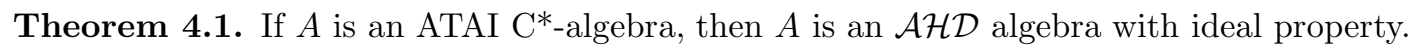

Proof. By remark 3.10, $A$ has the ideal property. So we will only prove $A$ is an $\mathcal{A H D}$ algebra.

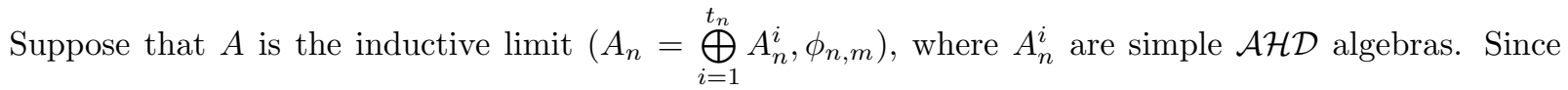
all $A_{n}^{i}$ are simple, without lose of generality, we can assume that all the homorphisms $\phi_{n, m}$ are injective. We will construct a sequence of sub-C ${ }^{*}$-algebras $B_{n}^{i} \subset A_{n}^{i}$ which are direct sums of $\mathcal{H D}$ building blocks and homomorphisms $\psi_{n, n+1}: B_{n}=\bigoplus B_{n}^{i} \rightarrow B_{n+1}=\bigoplus B_{n+1}^{i}$ such that the diagram

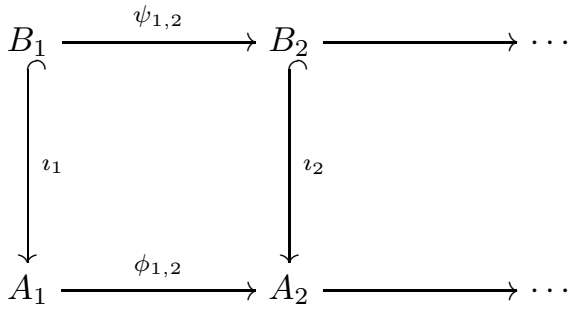

is approximately commutative in the sense of Elliott and $\overline{\bigcup_{n=1}^{\infty} \phi_{n, \infty}\left(\imath_{n}\left(B_{n}\right)\right)}=A$.

Let $\left\{a_{n, k}\right\}_{k=1}^{\infty}$ be a dense subset of the unit ball of $A_{n}$ and $\varepsilon_{n}=\frac{1}{2^{n}}$. Let $G_{1} \subset A_{1}$ be defined by $G_{1}=\bigoplus_{i=1}^{t_{1}}\left\{\pi_{i}\left(a_{11}\right)\right\} \subset \bigoplus_{i=1}^{t_{1}} A_{1}^{i}=A_{1}$ where $\pi_{1}: A_{1} \rightarrow A_{1}^{i}$ are canonical projections.

Fix $i \in\left\{1,2, \cdots, t_{1}\right\}$, one can write $A_{1}^{i}=\lim _{n \rightarrow \infty}\left(C_{n}, \xi_{n, m}\right)$ as in Lemma 3.6 with injective homomorphisms $\xi_{n, m}$. 
For $\pi_{i}\left(G_{1}\right) \subset A_{1}^{i}$, we choose $n$ large enough such that $\pi_{i}\left(G_{1}\right) \subset_{\varepsilon_{1}} \xi_{n, \infty}\left(C_{n}\right)$. And let $B_{1}^{i}=C_{n}$ and $F_{1}^{i}$ a finite set with $\pi_{i}\left(G_{1}\right) \subset_{\varepsilon_{1}} \imath\left(F_{1}^{i}\right)$, where $\imath$ is the inclusion homomorphism $\xi_{n, \infty}$. Let $B=\bigoplus_{i=1}^{t_{1}} B_{1}^{i} \hookrightarrow \bigoplus_{i=1}^{t_{1}} A_{1}^{i}$, and $F_{1}=\bigoplus F_{1}^{i}$.

We will construct the following diagram

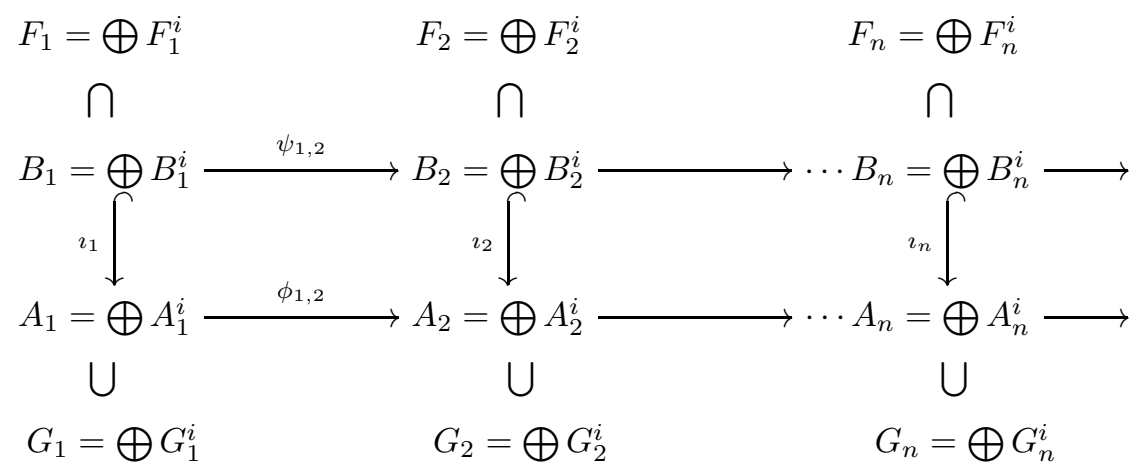

such that

(1) $G_{k} \subset_{\varepsilon_{k}} \imath_{k}\left(F_{k}\right), G_{k} \supset \phi_{k-1, k}\left(G_{k-1} \cup\left\{a_{k-1, k}\right\}\right) \cup\left\{a_{k, j}\right\}_{j=1}^{k}$;

(2) $\left\|\imath_{k} \circ \psi_{k-1, k}(f)-\phi_{k-1, k} \circ \imath_{k-1}(f)\right\|<\varepsilon_{k-1}, \quad \forall f \in F_{k-1}$.

The construction will be carried out by induction. Suppose that we have the diagram until $F_{n} \subset B_{n} \hookrightarrow$ $A_{n} \supset G_{n}$. We will construct the next piece of the diagram.

Let $P^{i, j}=\phi_{n, n+1}^{i, j}\left(\mathbf{1}_{A_{n}^{i}}\right) \in A_{n+1}^{j}$. Then $\left\{P^{i, j}\right\}_{i=1}^{t_{n}}$ is a set of mutually orthogonal projections in $A_{n+1}^{j}$. Apply Corollary 3.7 for $A_{n+1}^{j}$ in place of $A$ and $\left\{P^{i, j}\right\}_{i=1}^{t_{n}}$ in places of $\left\{P_{1}, P_{2}, \cdots, P_{k}\right\}$, we can write $A_{n+1}^{j}=$ $\lim \left(C_{k}, \lambda_{k, l}\right)$ with $P^{i, j} A_{n+1}^{j} P^{i, j}=\lim _{k}\left(Q_{k}^{i, j} C_{k} Q_{k}^{i, j},\left.\lambda_{k, l}\right|_{Q_{k}^{i, j} C_{k} Q_{k}^{i, j}}\right)\left(\right.$ where $\lambda_{k, l}\left(Q_{k}^{i, j}\right)=Q_{l}^{i, j} \in C_{l}$ ) being $\mathcal{A H \mathcal { D }}$ inductive limit algebra as described in Lemma 3.6. (Warning: Do not confuse these $C_{k}$ with the $C_{n}$ in the expression $A_{1}^{i}=\lim _{n \rightarrow \infty}\left(C_{n}, \xi_{n, m}\right)$.)

For each pair $i, j$, we apply Lemma 3.8 to the homomorphism $\phi_{n, n+1}^{i, j}: A_{n}^{i} \rightarrow P^{i, j} A_{n+1}^{j} P^{i, j}$ in place of $\Lambda: A \rightarrow A^{\prime}$, and $F_{n}^{i} \subset B_{n}^{i}$ in place of $F \in A_{m}$, there is an $l$ (large enough) and a homomorphism $\psi_{n, n+1}^{i, j}: B_{n}^{i} \rightarrow Q_{l}^{i, j} C_{l} Q_{l}^{i, j}$ such that

$$
\left\|\phi_{n, n+1}^{i, j} \circ \imath_{n}(f)-\imath_{n+1} \circ \psi_{n, n+1}^{i, j}(f)\right\|<\varepsilon \quad \forall f \in F_{n}^{i},
$$

where $\left.\imath_{n+1}\right|_{Q_{l}^{i, j} C_{l} Q_{l}^{i, j}}=\left.\lambda_{l, \infty}\right|_{Q_{l}^{i, j} C_{l} Q_{l}^{i, j}}$.

Finally choose $G_{n+1}^{j} \supset \pi_{j}\left[\left(G_{n} \cup\left\{a_{n, n+1}\right\}\right) \cup\left\{a_{n+1, j}\right\}_{j=1}^{n+1} \cup \imath_{n+1}\left(\bigcup_{k} \psi_{n, n+1}^{i, j}\left(F_{n}^{i}\right)\right)\right]$ and $G_{n+1}=\bigoplus G_{n+1}^{j}$. By increasing $l$, we can assume that there is an $F_{n+1}^{\prime j} \subset C_{l}$ such that $G_{n+1}^{j} \subset_{\varepsilon_{n+1}} F_{n+1}^{\prime j}$. Define $B_{n+1}^{j}$ to be $C_{l}$ which is a subalgebra of $A_{n+1}^{j}$. Let $F_{n+1}^{j} \subset B_{n+1}^{j}$ be defined by $F_{n+1}^{j}=F_{n+1}^{\prime j} \cup\left(\bigoplus_{i} \phi_{n, n+1}^{i, j}\left(F_{n}^{i}\right)\right)$ and let $F_{n+1}=\bigoplus F_{n+1}^{j}$. This ends our inductive construction. By the Elliott intertwining argument, $\lim \left(B_{n}, \psi_{n, m}\right)=\lim \left(A_{n}, \phi_{n, m}\right)$. This ends the proof. 
Theorem 4.2. Let $A=\lim \left(A_{n}, \phi_{n, m}\right)$ and $B=\lim \left(B_{n}, \psi_{n, m}\right)$ be inductive limit algebras with $A_{n}^{i}, B_{n}^{i}$ being unital separable nuclear simple TAI algebras with UCT. Suppose that there is an isomorphism

$$
\alpha:\left(\underline{K}(A), \underline{K}(A)^{+}, \sum A\right) \longrightarrow\left(\underline{K}(B), \underline{K}(B)^{+}, \sum B\right)
$$

which is compatible with Bockstein operations. Suppose that for each projection $p \in A$ and $\bar{p} \in B$ with $\alpha([p])=[\bar{p}]$, there exist a unital positive linear isomorphism

$$
\xi^{p, \bar{p}}: \operatorname{AffT}(p A p) \longrightarrow \operatorname{AffT}(\bar{p} B \bar{p})
$$

and an isometric group isomorphism

$$
\gamma^{p, \bar{p}}: U(p A p) / \widetilde{S U}(p A p) \longrightarrow U(\bar{p} B \bar{p}) / \widetilde{S U}(\bar{p} B \bar{p})
$$

satisfying the following compatibility conditions:

(1) For each pair of projections $p<q<\in A$ and $\bar{p}<\bar{q}<\in B$ with $\alpha([p])=[\bar{p}], \alpha([q])=[\bar{q}]$, the diagrams

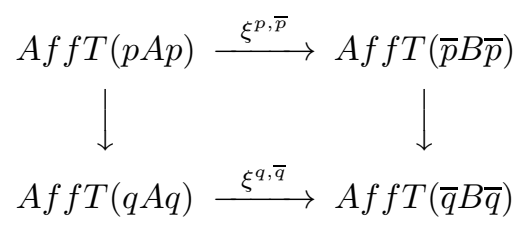

and

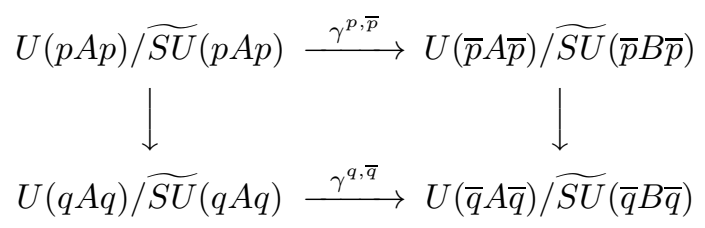

commute, where the vertical maps are induced by the inclusion homomorphisms.

(2) The maps $\alpha_{0}$ and $\xi^{p, \bar{p}}$ are compatible, that is, the diagram

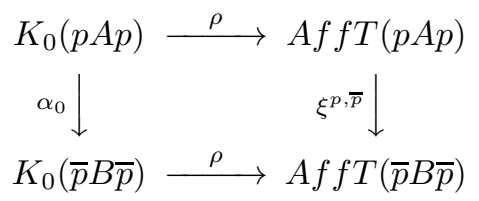

commutes (this is not an extra requirement, since it follows from the commutativity of the first diagram in (1) above by [Ji-Jiang]), and then we have the map (still denoted by $\xi^{p, \bar{p}}$ ):

$$
\xi^{p, \bar{p}}: \operatorname{AffT}(p A p) / \widetilde{\rho K_{0}}(p A p) \longrightarrow \operatorname{AffT}(\bar{p} B \bar{p}) / \widetilde{\rho K_{0}}(\bar{p} B \bar{p})
$$

(3) The map $\xi^{p, \bar{p}}$ and $\gamma^{p, \bar{p}}$ are compatible, that is, the diagram

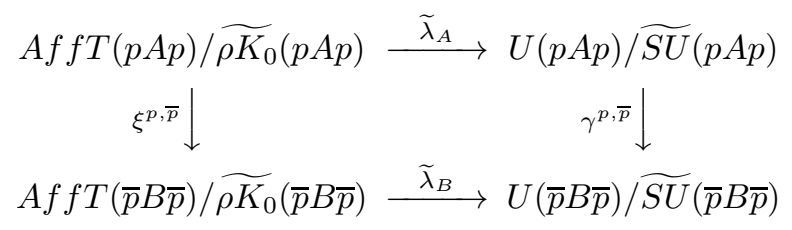

commutes.

(4) The map $\alpha_{1}: K_{1}(p A p) / \operatorname{tor} K_{1}(p A p) \longrightarrow K_{1}(\bar{p} B \bar{p}) / \operatorname{tor} K_{1}(\bar{p} B \bar{p})$ (note that $\alpha$ keeps the positive cone of $\underline{K}(A)^{+}$and therefore takes $K_{1}(p A p) \subset K_{1}(A)$ to $K_{1}(\bar{p} B \bar{p}) \subset K_{1}(B)$ is compatible with $\gamma^{p, \bar{p}}$, that is, the 
diagram

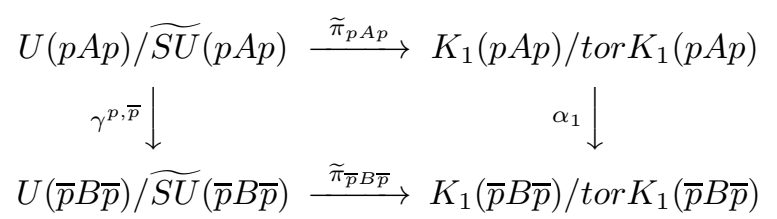

commutes.

Then there is an isomorphism $\Gamma: A \rightarrow B$ such that

(a) $K(\Gamma)=\alpha$, and

(b)If $\Gamma_{p}: p A p \rightarrow \Gamma(p) B \Gamma(p)$ is the restriction of $\Gamma$, then $\operatorname{Aff} T\left(\Gamma_{p}\right)=\xi^{p, \bar{p}}$ and $\Gamma_{p}^{\natural}=\gamma^{p, \bar{p}}$, where $[\bar{p}]=[\Gamma(p)]$.

Proof. It follows from the fact of Theorem 4.1 that all ATAI algebras are $\mathcal{A H D}$ algebras with ideal property and Proposition 3.11 ([GJL1]) that all $\mathcal{A H D}$ algebras with ideal property are classified by $\operatorname{Inv}(A)$.

Corollary 4.3. (A)([Jiang1], Theorem 3.11) Let $A=\lim _{n \rightarrow \infty}\left(A_{n}=\bigoplus A_{n}^{i}, \phi_{n, m}\right)$ and $B=\lim _{n \rightarrow \infty}\left(B_{n}=\right.$ $\left.\bigoplus B_{n}^{i}, \psi_{n, m}\right)$ be inductive limits with $A_{n}^{i}, B_{n}^{i}$ being unital separable nuclear simple TAI algebras with UCT and torsion $K_{1}$-group. Assume that there is an isomorphism $\alpha \in H_{0} m_{\Lambda}(\underline{K}(A), \underline{K}(B))$ such that

$$
\alpha\left(\underline{K}(A)^{+}\right)=\underline{K}(B)^{+}, \quad \alpha\left(\sum A\right)=\sum B .
$$

and for each pair of projections $p \in A, q \in B$ with $\alpha([p])=[q]$, there is a continuous affine homomorphism

$$
\xi^{p, q}: T(q B q) \rightarrow T(p A p)
$$

which is compatible in the sense of 2.19 , then there is an isomorphism $\Lambda: A \rightarrow B$ which induces $\alpha$ and $\xi$ above.

(B) ([Fa]) Let $A$ and $B$ be two ATAF algebras. Suppose that is an isomorphism of ordered groups

$$
\alpha:\left(\underline{K}(A), \underline{K}(A)^{+}, \sum A\right) \rightarrow\left(\underline{K}(B), \underline{K}(B)^{+}, \sum B\right)
$$

which preserves the action of the Bockstein operations. Then there is a $*$-isomorphism $\varphi: A \rightarrow B$ with $\varphi_{*}=\alpha$.

Proof. (A) It follows from the fact that $K_{1}(A)=\operatorname{tor} K_{1}(A), K_{1}(B)=\operatorname{tor} K_{1}(B)$ and Proposition 3.10 ([GJL2, Proposition 2.38]).

(B) From Theorem 4.1, if $A$ is an ATAF $\mathrm{C}^{*}$-algebra, then $A$ is an $\mathcal{A H \mathcal { D }}$ algebra of real rank zero which is classified in $[\mathrm{DG}]$ (Note that $\mathcal{A H D}$ algebra in $[\mathrm{DG}]$ is denoted by ASH algebra). 


\section{References}

[1] [D] M. Dadarlat, Reduction to dimension three of local spectra of Real rank zero C*-algebras, J. Reine Angew. Math. 460(1995) 189-212

[2] [DG] M. Dadarlat and G. Gong, A classification result for approximately homogeneous C*-algebras of real rank zero, Geometric and Functional Analysis, 7(1997) 646-711

[3] [Ell1] G. A. Elliott, On the classification of C*-algebras of real rank zero, J. Reine Angew. Math. 443(1993) 263-290

[4] [El12] G. A. Elliott, A classification of certain simple $\mathrm{C}^{*}$-algebras, Quantum and Non-Commutative Analysis, Kluwer, Dordrecht, (1993), pp, 373-388

[5] [El13] G. A. Elliott, A classification of certain simple C*-algebras, II, J. Ramaunjan Math. Soc. 12 (1997), 97-134

[6] [EE] G. A. Elliott, D. E. Evans: The structure of the irrational rotation C*-algebra. Ann. of Math. (2) 138 (1993), no. 3, 477-501.

[7] [EG1] G. A. Elliott and G. Gong, On the inductive limits of matrix algebras over two-tori, American. J. Math 118(1996) 263-290

[8] [EG2] G. A. Elliott and G. Gong, On the classification of C*-algebras of real rank zero, II. Ann. of Math 144(1996) 497-610

[9] [EGL1] G. A. Elliott, G. Gong and L. Li, On the classification of simple inductive limit C*-algebras, II; The isomorphism Theorem, Invent. Math. 168(2)(2007) 249-320

[10] [EGL2] G. A. Elliott, G. Gong and L. Li, Injectivity of the connecting maps in AH inductive limit systems, Canand. Math. Bull. 26(2004) 4-10

[11] [EGJS] G. A. Elliott, G. Gong, X. Jiang, H. Su: Aclassification of simple limits of dimension drop C*-algebras. Fields Inst. Commun. 13, 125-143 (1997)

[12] [EGLP] G. A. Elliott, G. Gong, H. Lin, C.Pasnicu: Abelian $C^{*}$-subslgebras of $C^{*}$-algebras of real rank zero and inductive limit C*-algebras. Duke Math. J. 83, 511-554 (1996)

[13] [EGS] G. A. Elliott, G. Gong, H. Su: On the classification of $\mathrm{C}^{*}$-algebras of real rank zero, IV: Reduction to local spectrum of dimension two. Fields Inst. Commun. 20, 73-95 (1998)

[14] [Fa] X. C. Fang, The classification of certain non-simple $\mathrm{C}^{*}$-algebras of tracial rank zero, J. Funct. Anal. 256(2009) 38613891

[15] [G1-2] G. Gong, On inductive limit of matrix algebras over higher dimension spaces, Part I, II, Math Scand. 80(1997) 45-60, 61-100

[16] [G3] G. Gong, On the classification of simple inductive limit $\mathrm{C}^{*}$-algebras, I: Reduction Theorems. Doc. Math. 7(2002) $255-461$

[17] [GJL1] G. Gong, C. Jiang, L. Li, A classification of inductive limit C*-algebras with ideal property, Preprint,

[18] [GJL2] G. Gong, C. Jiang, L. Li, Hausdorffized algebraic $K_{1}$ group and C*-algebras with the ideal property, Preprint,

[19] [GJLP1] G. Gong, C. Jiang, L. Li, C. Pasnicu, AT structure of AH algebras with ideal property and torsion free K-theory, J. Func. Anal. 58(2010) 2119-2143

[20] [GJLP2] G. Gong, C. Jiang, L. Li, C. Pasnicu, A Reduction theorem for AH algebras with the ideal property, Int Math Res Notices. 24(2018), 7606-7641

[21] [GL] G. Gong and H. Lin, Almost multiplicative morphisms and K-theory, International J. Math. 11 (2000) 983-1000.

[22] [GLN] G. Gong, H. Lin and Z. Niu, Classification of finite simple amenable $\mathcal{Z}$-stable $\mathrm{C}^{*}$-algebras, Preprint arXiv:1501.00135

[23] [GLX] G. Gong, H. Lin and Y. Xue, Determinant rank of C*-algebras, Pacific J. Math 274 (2015) No. 2 405-436

[24] [Ji-Jiang] K. Ji and C. Jiang, A complete classification of AI algebra with ideal property, Canadian. J. Math, 63(2), (2011), 381-412

[25] [Jiang1] C. Jiang, A classification of non simple $\mathrm{C}^{*}$-algebras of tracial rank one: Inductive limit of finite direct sums of simple TAI C*-algebras, J. Topol. Anal. 3 No.3(2011), 385-404

[26] [Jiang2] C. Jiang, Reduction to dimension two of local spectrum for AH algebras with ideal property, Canand. Math. Bull. 60(2017) No.4, 791-806

[27] [Li1] L. Li, On the classification of simple $\mathrm{C}^{*}$-algebras: Inductive limit of matrix algebras over trees, Mem Amer. Math, Soc. 127(605) 1997 
[28] [Li2] L. Li, Simple inductive limit $\mathrm{C}^{*}$-algebras: Spectra and approximation by interval algebras, J. Reine Angew Math 507(1999) 57-79

[29] [Li3] L. Li, Classification of simple $\mathrm{C}^{*}$-algebras: Inductive limit of matrix algebras over 1-dimensional spaces, J. Func. Anal. 192(2002) 1-51

[30] [Li4] L. Li, Reduction to dimension two of local spectrum for simple AH algebras, J. of Ramanujian Math. Soc. 21 No.4(2006) 365-390

[31] [Lin1] H. Lin, Tracially AF C*-algebras, Trans. Amer. Math. Soc. 353 (2001) No. 2, 693-722

[32] [Lin2] H. Lin, Simple nuclear $C^{*}$-algrbras of tracial topological rank one. J. Funct. Anal. 251(2007), No. 2, 601-679

[33] [NT] K. E. Nielsen and K. Thomsen, Limits of circle algebras. Expo. Math. 14, 17-56 (1996)

[34] [Popa] S. Popa, On locally finite-dimensional approximation of $\mathrm{C}^{*}$-algebras, Pacifi J. Math. (1997) 141-158

[35] [Rob] L. Robert, Classification of inductive limits of 1-dimensional NCCW-complexes, Adv. in Math. 231(2012), 2802-2836

[36] [R] M. Rordam, Classification of certain infinite simple C*-algebras. J. Funct. Anal. 131(1995), 415-458

[37] [Stev] K. Stevens, The classification of certain non-simple approximate interval algebras. Fields Inst. Commun. 20(1998) 105-148

[38] [Thm1] K. Thomsen, Insuctive limit of interval algebras, American J. of Math 116, 605C620 (1994)

[39] [Thm2] K. Thomsen, Limits of certain subhomogeneous C*-algebras, Mem. Soc. Math. Fr. (N.S.) 71(1999) 